\title{
Effect of anisotropic impurity scattering in superconductors
}

\author{
Grzegorz Harańt and A. D. S. Nagi \\ Department of Physics, University of Waterloo, Waterloo, Ontario, Canada, N2L 3G1
}

(August 30, 2021)

\begin{abstract}
We discuss the weak-coupling BCS theory of a superconductor with the impurities, accounting for their anisotropic momentum-dependent potential. The impurity scattering process is considered in the t-matrix approximation and its influence on the superconducting critical temperature $T_{c}$ is studied in the Born and unitary limit for a $d_{x^{2}-y^{2-}}$ and $\left(d_{x^{2}-y^{2}}+s\right)$-wave superconductors. We observe a significant dependence of the pair-breaking strength on the symmetry of the scattering potential and classify the impurity potentials according to their ability to alter $T_{c}$. A good agreement with the experimental data for Zn doping and oxygen irradiation in the overdoped cuprates is found.

PACS numbers: 74.20.-z, 74.62.-c, 74.62.Dh
\end{abstract}

Typeset using REVTEX 


\section{INTRODUCTION}

Several experiments probing the effect of impurities or lattice defects on superconductivity in the cuprates have been carried out 22 in order to get more insight into the symmetry of the superconducting state. The most thoroughly studied defects are $\mathrm{Zn}$ and $\mathrm{Ni}$ substitutions 18 on the planar $\mathrm{Cu}$ sites and irradiation induced oxygen vacancies 22 in the copper oxygen planes. Yet the results are difficult to explain within a standard AbrikosovGorkov type theory of impurity scattering 23 for the scenario of the $d$-wave superconductivity, which is predicted to be extremely suppressed by the impurities.24 27 This issue was critically examined by Radtke et al.24 who considered isotropic nonmagnetic impurity scattering in the Born approximation and obtained the critical temperature in both weak- and strong-coupling approach close to the Abrikosov-Gorkov scaling function. A comparison to the electron irradiation data 19 in $\mathrm{YBa}_{2} \mathrm{Cu}_{3} \mathrm{O}_{7-\delta}(Y-123)$ showed that the theoretically predicted $\mathrm{T}_{c}$ was about twice as much reduced by the impurities than observed. This inconsistency can be settled down within the weak impurity scattering model if the impurity scattering rate a factor of 3 less than the one deduced from the transport measurements is assumed20.21 which is equivalent to an introduction of two separate relaxation time scales - one defining the scattering time in pair-breaking processes and the other representing the transport scattering time. Such a distinction occurs naturally as a consequence of an impurity momentum-dependent scattering probability. 28 The issue of a possible anisotropy in the impurity scattering potential was suggested in a discussion of the irradiation data by Giapintzakis et al 19 where the authors evoked a model by Millis et al.29 A more general formulation of the problem was given in our previous paper, 30 where the effective correlation between two impurity vertex functions was assumed in the form $\left|w_{0}\right|^{2}+\left|w_{1}\right|^{2} f(\mathbf{k}) f\left(\mathbf{k}^{\prime}\right)$, with $\left|w_{0}\right|$ and $\left|w_{1}\right|$ representing isotropic and anisotropic impurity scattering amplitude respectively and $f(\mathbf{k})$ determining

the symmetry of the impurity potential. 31 Our analysis showed that the symmetry of the anisotropic potential is an important factor and a significant reduction in the pair-breaking strength appears for large values of $\langle e f\rangle^{2}=\left[\int_{F S} d S_{k} n(\mathbf{k}) e(\mathbf{k}) f(\mathbf{k})\right]^{2}$, where $\int_{F S} d S_{k}$ denotes 
integration over the Fermi surface (FS) and $n(\mathbf{k})$ is the normalized $\left(\int_{F S} d S_{k} n(\mathbf{k})=1\right)$ angleresolved FS density of states. Particularly low $T_{c}$ suppression is predicted for $\langle e f\rangle^{2}=1$ that is for the anisotropy of the scattering potential in phase with the order parameter, which in the case of the $d_{x^{2}-y^{2}}$-wave superconductor corresponds to $f(\mathbf{k}) \sim k_{x}^{2}-k_{y}^{2}$. Within this approach we were also able to understand quantitatively the irradiation data 19 in $Y-123$.

Through the postulation of the analytic form of the square value of the impurity potential this approach has been designed for the second-order Born approximation and cannot be efficiently extended to include multiple impurity scattering processes. Such a generalization is important from the theoretical and experimental points of view, especially in the light of the recent experiments suggesting a possible strong (close to unitary) scattering by $\mathrm{Zn}$ atoms in $\mathrm{Y}-123$ and $\mathrm{La}_{2-x} \mathrm{Sr}_{x} \mathrm{CuO}_{4}(\mathrm{La}-214)$ compounds. $\mathrm{Z}$ For that purpose a model based on the assumption of the impurity potential and not its square value is needed.

In this paper we study in the t-matrix approximation the pair-breaking effect of the nonmagnetic impurities with the anisotropic momentum-dependent factorizable potential. The influence on the superconducting transition temperature is analyzed quantitatively in the Born and unitary scattering limits. In particular, we find that the scattering from impurity potential in phase with the order parameter leads to a stronger suppression of the critical temperature than from the impurity potential orthogonal to the superconducting state. On the other hand, the superconducting state appears very robust to the potential scattering with its maxima in the region of the nodes of the order parameter. Finally, we find that the $\mathrm{Zn}$ and electron irradiation $T_{c}$ suppression data in $Y-123$ and $L a-214$ are in the range predicted by our model.

This work is organized as follows. In Sec. II, we introduce the anisotropic momentumdependent impurity potential. In Sec. III, we derive the expressions necessary for the analysis of the scattering process of arbitrary strength. In Sec. IV, we obtain the selfenergies due to impurity scattering at the superconducting-normal state phase transition which allows the evaluation of the critical temperature. Then we examine analytically Born and unitary scattering limits. In Sec. V, we calculate numerically the critical temperature 
for $d$ - and $(d+s)$-wave superconducting states in the presence of impurities accounting for their anisotropic potential of $p-, d-, f_{-}$, and $g$-wave symmetry in Born and unitary limit. In Sec. VI, we compare the results with data on $\mathrm{Zn}$ substituted and irradiated overdoped La-214 and optimally doped $Y-123$ samples. In Sec. VII, we present our conclusions. Except for comparing to the experimental data we assume $\hbar=k_{B}=1$ in the calculations.

\section{IMPURITY SCATTERING POTENTIAL}

As the impurity scattering strength is rather impossible to be determined from first principles, the information about the scattering process is usually deduced from a comparison of the experimental data with the theoretical models. These models assume a certain phenomenological impurity potential23,24,29,30,32 36 which is verified by a fit to the available data. We proceed in the same way by analyzing the impurity scattering potential of the factorizable form

$$
v\left(\mathbf{k}, \mathbf{k}^{\prime}\right)=v_{i}+v_{a} f(\mathbf{k}) f\left(\mathbf{k}^{\prime}\right)
$$

The above interaction consists of two channels - the isotropic scattering channel with the scattering amplitude $v_{i}$, and the anisotropic one determined by the scattering strength $v_{a}$ and momentum-dependent function $f(\mathbf{k})$. We assume that the $f(\mathbf{k})$ average value over the Fermi surface $\langle f\rangle=\int_{F S} d S_{k} n(\mathbf{k}) f(\mathbf{k})=0$. Therefore, $f(\mathbf{k})$ is orthogonal to the isotropic s-wave term in a sense of a scalar product defined as the FS integral and in consequence a symmetry other than the identity is introduced into the impurity potential. An additional normalization $\left\langle f^{2}\right\rangle=1$ gives $v_{a}$ the meaning of the scattering strength magnitude in the anisotropic channel. The chosen potential depends on the absolute orientations in the crystal of the incoming and outgoing (scattered) particles momenta, not only on the angle

between $\mathbf{k}$ and $\mathbf{k}^{\prime}$. The usually assumed isotropic conditions 37 of a spherical (cylindrical in two dimensions) constant energy surface and the scattering probability dependent only on the angle of deflexion are broken here. 38 This leads to a momentum-dependence of the 
time between scattering events determined by the imaginary part of the self-energy and a momentum-dependent relaxation time in the Boltzmann equation. 99 . This second quantity is worth mentioning as it provides the information about the normal state transport properties and can be used as an additional physical assessment of the phenomenological model.28 A constraint on the potential (1) which follows immediately from the analysis of the normal state properties is a nonzero value of the scattering amplitude in the isotropic channel $v_{i}$. A lack of the s-wave scattering may lead in some cases to an infinite value of some elements in the dc conductivity tensor, 28 that is to nonphysical transport properties.

The anisotropic potential from Eq. (1) distinguishes a certain set of coordinates which we think should coincide with the main directions in the crystal. This assumption seems rather plausible because the impurity potential is determined by the dopant atom itself as well as its substitution site in the crystal. The potential produced by a given sort of impurity (defect) may be considered unique since the impurities tend to selectively substitute at characteristic sites in the crystal. The $\mathrm{Zn}$ and $\mathrm{Ni}$ atoms occupy the in-plane $\mathrm{Cu}$ sites 18 and the electron irradiation displaces the oxygen atoms from the $\mathrm{CuO}_{2}$ planes. 1922 Therefore on a short length scale of the order of magnitude of the lattice constant a given impurity dopant produces the same potential of the same orientation throughout the crystal. On the other hand, on a large length scale the impurity distribution in the system is random and the Abrikosov-Gorkov's methode3 of averaging the Green's functions is applicable.

We have not made any assumption about the electron energy band yet and we use a general formalism deriving the equations valid for an arbitrary Fermi surface. For the computational simplicity, however, the numerical results are obtained for a cylindrical FS. This approximation allows to study the effect of the anisotropy of the impurity potential alone. The anisotropy of the Fermi surface may equally enhance or suppress the pairbreaking effect of the impurities. The detailed quantitative calculations are needed to answer this question which is beyond the scope of the present paper.

For the numerical calculations we take the function $f(\mathbf{k})$ in the scattering potential (11) proportional to the harmonic functions which in a polar angle notation read $\sin (l \phi)$ and 
$\cos (l \phi)$, where $l$ is an integer number. Therefore we study the effect of the basis elements in a space of the functions determined by a two-dimensional momentum vector.

\section{T-MATRIX APPROXIMATION FOR THE SELF-ENERGY}

We study the effect of potential scattering by spinless, noninteracting impurities on the single-particle propagator for superconducting electrons

$$
\hat{G}(\mathbf{k}, \omega)=\left[i \omega \hat{\tau}_{0} \hat{\sigma}_{0}-\xi_{k} \hat{\tau}_{3} \hat{\sigma}_{0}-\Delta(\mathbf{k}) i \hat{\tau}_{2} \hat{\sigma}_{2}-\hat{\Sigma}(\mathbf{k}, \omega)\right]^{-1}
$$

Here $\xi_{k}$ is the quasiparticle energy, $\omega=\pi T(2 m+1)$, where $\mathrm{T}$ is the temperature and $m$ is an integer. $\hat{\tau}_{j}, \hat{\sigma}_{j}(j=1,2,3)$ are the Pauli matrices and $\hat{\tau}_{0}, \hat{\sigma}_{0}$ are the unit matrices in particle-hole (Nambu) and spin space respectively. The order parameter $\Delta(\mathbf{k})$ is defined as

$$
\Delta(\mathbf{k})=\Delta e(\mathbf{k})
$$

where $e(\mathbf{k})$ is a momentum-dependent real function which may belong to a one-dimensional (1D) irreducible representation of the crystal point group or may be given by a linear combination of the basis functions of different 1D representations. We normalize $e(\mathbf{k})$ by taking

its average value over the Fermi surface $\left\langle e^{2}\right\rangle=1$. This normalization gives $\Delta$ the meaning of the magnitude of the order parameter. The self-energy $\hat{\Sigma}(\mathbf{k}, \omega)$ and consequently the Green's function (Eq. (21) have been obtained by applying Abrikosov-Gorkov's technique 3 of averaging over the coordinates of the impurities and depend on only one momentum vector $\mathbf{k}$. In this approximation

$$
\hat{\Sigma}(\mathbf{k}, \omega)=n \hat{T}(\mathbf{k}, \mathbf{k}, \omega)
$$

where $n$ is the impurity concentration and $\hat{T}$ obeys the Lippmann-Schwinger equation 3 , 近, 国

$$
\hat{T}\left(\mathbf{k}, \mathbf{k}^{\prime}, \omega\right)=\hat{v}\left(\mathbf{k}, \mathbf{k}^{\prime}\right)+\sum_{\mathbf{k}^{\prime \prime}} \hat{v}\left(\mathbf{k}, \mathbf{k}^{\prime \prime}\right) \hat{G}\left(\mathbf{k}^{\prime \prime}, \omega\right) \hat{T}\left(\mathbf{k}^{\prime \prime}, \mathbf{k}^{\prime}, \omega\right)
$$

Since the scattering potential for a single electron 


$$
\hat{v}\left(\mathbf{k}, \mathbf{k}^{\prime}\right)=v\left(\mathbf{k}, \mathbf{k}^{\prime}\right) \hat{\tau}_{3} \hat{\sigma}_{0}
$$

is momentum-dependent (Eq. (1)), the vertex part $\hat{T}\left(\mathbf{k}, \mathbf{k}^{\prime}, \omega\right)$ is a function of two momenta $\mathbf{k}$ and $\mathbf{k}^{\prime}$. Therefore it should be evaluated from Eq. (5) using the explicit form of $v\left(\mathbf{k}, \mathbf{k}^{\prime}\right)$ first, and then the self-energy can be obtained according to Eq. (四) by taking $\mathbf{k}^{\prime}=\mathbf{k}$. We proceed to a solution by defining

$$
\hat{g}_{i}(\omega)=\sum_{\mathbf{k}} f^{i}(\mathbf{k}) \hat{G}(\mathbf{k}, \omega), \quad i=0,1,2
$$

and expanding all matrix quantities as

$$
\begin{gathered}
\hat{\Sigma}(\mathbf{k}, \omega)=\Sigma_{0}(\mathbf{k}, \omega) \hat{\tau}_{0} \hat{\sigma}_{0}+\Sigma_{1}(\mathbf{k}, \omega) \hat{\tau}_{1} \hat{\sigma}_{2}+\Sigma_{2}(\mathbf{k}, \omega) \hat{\tau}_{2} \hat{\sigma}_{2}+\Sigma_{3}(\mathbf{k}, \omega) \hat{\tau}_{3} \hat{\sigma}_{0} \\
\hat{g}_{i}(\omega)=g_{i 0}(\omega) \hat{\tau}_{0} \hat{\sigma}_{0}+g_{i 1}(\omega) \hat{\tau}_{1} \hat{\sigma}_{2}+g_{i 2}(\omega) \hat{\tau}_{2} \hat{\sigma}_{2}+g_{i 3}(\omega) \hat{\tau}_{3} \hat{\sigma}_{0}
\end{gathered}
$$

The expression for the one-particle Green's function is then

$$
\hat{G}(\mathbf{k}, \omega)=-\frac{i \tilde{\omega} \hat{\tau}_{0} \hat{\sigma}_{0}+\tilde{\xi}_{k} \hat{\tau}_{3} \hat{\sigma}_{0}+\tilde{\Delta}(\mathbf{k}) i \hat{\tau}_{2} \hat{\sigma}_{2}+\tilde{\Delta}^{\prime}(\mathbf{k}) \hat{\tau}_{1} \hat{\sigma}_{2}}{\tilde{\omega}^{2}+\tilde{\xi}_{k}^{2}-\tilde{\Delta}^{2}(\mathbf{k})+\tilde{\Delta}^{\prime 2}(\mathbf{k})}
$$

with $\tilde{\omega}=\omega+i \Sigma_{0}(\mathbf{k}, \omega), \tilde{\xi}_{k}=\xi_{k}+\Sigma_{3}(\mathbf{k}, \omega), \tilde{\Delta}(\mathbf{k})=\Delta(\mathbf{k})-i \Sigma_{2}(\mathbf{k}, \omega)$ and $\tilde{\Delta}^{\prime}(\mathbf{k})=\Sigma_{1}(\mathbf{k}, \omega)$. The formal solutions for the self-energies $\Sigma_{j}(\mathbf{k}, \omega)(j=0,1,2,3)$ obtained through Eqs. (雨) and (5) read

$$
\begin{aligned}
& \Sigma_{j}(\mathbf{k}, \omega)=s_{j}\left(v_{i}, v_{a}, \hat{g}_{0}, \hat{g}_{1}, \hat{g}_{2}\right) u\left(v_{i}, v_{a}, \hat{g}_{0}, \hat{g}_{1}, \hat{g}_{2}\right) \\
& +s_{j}\left(v_{a}, v_{i}, \hat{g}_{2}, \hat{g}_{1}, \hat{g}_{0}\right) u\left(v_{a}, v_{i}, \hat{g}_{2}, \hat{g}_{1}, \hat{g}_{0}\right) f^{2}(\mathbf{k}) \\
& +\left[t_{3-j}\left(v_{i}, v_{a}, \hat{g}_{0}, \hat{g}_{1}, \hat{g}_{2}\right)+t_{3-j}\left(v_{a}, v_{i}, \hat{g}_{2}, \hat{g}_{1}, \hat{g}_{0}\right)\right] f(\mathbf{k})
\end{aligned}
$$

Note a permutation: $v_{i} \leftrightarrow v_{a}, \hat{g}_{0} \leftrightarrow \hat{g}_{2}$, in the arguments of the anisotropic parts (proportional to $f(\mathbf{k})$ and $\left.f^{2}(\mathbf{k})\right)$ of $\Sigma_{j}(\mathbf{k}, \omega)$ in Eq. (11). The functions $s_{j}, t_{j}$ and $u$ are given by a series of equations

$$
\begin{aligned}
& s_{0}\left(v_{i}, v_{a}, \hat{g}_{0}, \hat{g}_{1}, \hat{g}_{2}\right)=a_{0} c_{3}+a_{1} c_{2}+a_{2} c_{1}+a_{3} c_{0} \\
& s_{1}\left(v_{i}, v_{a}, \hat{g}_{0}, \hat{g}_{1}, \hat{g}_{2}\right)=a_{0} c_{2}+a_{1} c_{3}+i a_{2} c_{0}-i a_{3} c_{1} \\
& s_{2}\left(v_{i}, v_{a}, \hat{g}_{0}, \hat{g}_{1}, \hat{g}_{2}\right)=a_{0} c_{1}-i a_{1} c_{0}+a_{2} c_{3}+i a_{3} c_{2} \\
& s_{3}\left(v_{i}, v_{a}, \hat{g}_{0}, \hat{g}_{1}, \hat{g}_{2}\right)=a_{0} c_{0}+i a_{1} c_{1}-i a_{2} c_{2}+a_{3} c_{3}
\end{aligned}
$$




$$
\begin{gathered}
t_{j}\left(v_{i}, v_{a}, \hat{g}_{0}, \hat{g}_{1}, \hat{g}_{2}\right)=v_{i} n c_{j}\left(c_{0}^{2}+c_{1}^{2}+c_{2}^{2}-c_{3}^{2}\right)^{-1} \\
u\left(v_{i}, v_{a}, \hat{g}_{0}, \hat{g}_{1}, \hat{g}_{2}\right)=v_{i} v_{a}^{-1} n d^{-1}\left(c_{0}^{2}+c_{1}^{2}+c_{2}^{2}-c_{3}^{2}\right)^{-1}
\end{gathered}
$$

with $d=-g_{10}^{2}+g_{11}^{2}+g_{12}^{2}+g_{13}^{2}$ and the coefficients $a_{j}, c_{j}(j=0,1,2,3)$ determined by twelve $g_{i k}$ elements (Eqs. (7) and (9)) given by the integrals of the products of the Green's function $\hat{G}(\mathbf{k}, \omega)$ and appropriate powers of the impurity potential anisotropy function $f(\mathbf{k})$. These coefficients are introduced in order to shorten and simplify the notation of the self-energy functions. We define them in the sequential formulas with a use of additional $b_{j}$ parameters

$$
\begin{gathered}
a_{0}=g_{13}\left(1-v_{a} g_{23}\right)-v_{a}\left(g_{12} g_{22}+g_{11} g_{21}-g_{10} g_{20}\right) \\
a_{1}=i g_{12}\left(1-v_{a} g_{23}\right)+v_{a}\left(i g_{13} g_{22}-g_{11} g_{20}+g_{10} g_{21}\right) \\
a_{2}=-i g_{11}\left(1-v_{a} g_{23}\right)-v_{a}\left(i g_{13} g_{21}+g_{12} g_{20}-g_{10} g_{22}\right) \\
a_{3}=-g_{10}\left(1-v_{a} g_{23}\right)-v_{a}\left(g_{13} g_{20}-i g_{12} g_{21}+i g_{11} g_{22}\right) \\
b_{0}=\left(1-v_{i} g_{03}\right) a_{0}+v_{i}\left(i g_{02} a_{1}-i g_{01} a_{2}-g_{00} a_{3}\right) \\
b_{1}=\left(1-v_{i} g_{03}\right) a_{1}+v_{i}\left(i g_{02} a_{0}+i g_{00} a_{2}+g_{01} a_{3}\right) \\
b_{2}=\left(1-v_{i} g_{03}\right) a_{2}+v_{i}\left(g_{02} a_{3}-i g_{01} a_{0}-i g_{00} a_{1}\right) \\
b_{3}=\left(1-v_{i} g_{03}\right) a_{3}-v_{i}\left(g_{02} a_{2}+g_{01} a_{1}+g_{00} a_{0}\right) \\
c_{0}=v_{a}^{-1} d^{-1} b_{0}-v_{i} g_{13} \\
c_{1}=i v_{a}^{-1} d^{-1} b_{1}-v_{i} g_{12} \\
c_{2}=-i v_{a}^{-1} d^{-1} b_{2}-v_{i} g_{11} \\
c_{3}=-v_{a}^{-1} d^{-1} b_{3}+v_{i} g_{10}
\end{gathered}
$$

The self-energies can be evaluated with a simultaneous solution of the gap equation

$$
\Delta(\mathbf{k}) i \hat{\sigma}_{2}=-T \sum_{\omega} \sum_{\mathbf{k}^{\prime}} \frac{1}{2} V_{\mathbf{k}, \mathbf{k}^{\prime}} \operatorname{tr}\left[\left(\hat{\tau}_{1}+\hat{\tau}_{2}\right) \hat{G}\left(\mathbf{k}^{\prime}, \omega\right)\right]
$$

where $V_{\mathbf{k}, \mathbf{k}^{\prime}}=-V_{0} e(\mathbf{k}) e\left(\mathbf{k}^{\prime}\right), V_{0}>0$, is the pair potential. The above lengthy expressions have been derived without any additional constraints and are fundamental to the considerations within the model. This generally complicated problem simplifies with the assumption 
of particle-hole symmetry of the excitation spectrum. It has been shown by Hirschfeld et

al 41 that in this case the $\hat{\tau}_{3}$ component of the integrated Green's function $\left(g_{03}\right)$ may be neglected in the presence of $s$-wave scatterers. Inclusion of higher-order angular momentum waves in the scattering potential makes this analysis considerably more difficult. In this paper, however, we calculate the self-energies at the phase transition and for that purpose we need to show only the consistency of the assumption $\Sigma_{3}=0$ at the critical temperature $T_{c}$. In the following we assume a particle-hole symmetry of the energy spectrum, take $g_{i 3}=0$ $(i=0,1,2)$ and check if this condition leads to a vanishing self-energy $\Sigma_{3}$.

\section{SELF-ENERGY AT PHASE TRANSITION}

We consider the effect of anisotropic impurity scattering on the critical temperature. At the superconducting-normal state phase transition the gap equation (18) transforms into

$$
1=V_{0} T_{c} \sum_{\omega} \sum_{\mathbf{k}} e(\mathbf{k}) \frac{e(\mathbf{k})+\frac{1}{i}\left(\Sigma_{1}(\mathbf{k}, \omega) / \Delta\right)_{\Delta=0}+\frac{1}{i}\left(\Sigma_{2}(\mathbf{k}, \omega) / \Delta\right)_{\Delta=0}}{\left(\omega+i \Sigma_{0}(\mathbf{k}, \omega)_{\Delta=0}\right)^{2}+\left(\xi_{k}+\Sigma_{3}(\mathbf{k}, \omega)_{\Delta=0}\right)^{2}}
$$

In order to find $T_{c}$ the self-energies in $\Delta \rightarrow 0$ limit are to be obtained. For the sake of convenience we introduce new parameters $c_{i}=1 /\left(\pi N_{0} v_{i}\right)$ and $c_{a}=1 /\left(\pi N_{0} v_{a}\right)$ describing the scattering strength in the isotropic $\left(c_{i}\right)$ and anisotropic $\left(c_{a}\right)$ channel respectively. $N_{0}$ represents the overall single-spin density of states at the Fermi level. A new measure of impurity concentration $\Gamma=n /\left(\pi N_{0}\right)$ is also used. Taking into account that $\left(g_{00}\right)_{\Delta=0}=$ $-i \pi N_{0} \operatorname{sgn}(\omega)$ and $\left(g_{20}\right)_{\Delta=0}=\left(g_{00}\right)_{\Delta=0}$ (because of normalization $\left\langle f^{2}\right\rangle=1$ ) we obtain

$$
\begin{gathered}
\Sigma_{0}(\mathbf{k}, \omega)_{\Delta=0}=-i \Gamma\left[\frac{1}{c_{i}^{2}+1}+\frac{1}{c_{a}^{2}+1} f^{2}(\mathbf{k})\right] \operatorname{sgn}(\omega) \\
\Sigma_{3}(\mathbf{k}, \omega)_{\Delta=0}=\Gamma\left[\frac{c_{i}}{c_{i}^{2}+1}+\frac{c_{a}}{c_{a}^{2}+1} f^{2}(\mathbf{k})\right]
\end{gathered}
$$

Calculation of $\left(\Sigma_{1}(\mathbf{k}, \omega) / \Delta\right)_{\Delta=0}$ and $\left(\Sigma_{2}(\mathbf{k}, \omega) / \Delta\right)_{\Delta=0}$ quantities is more tedious and requires a solution of two sets of two linear equations which determine four unknowns 
$\left(g_{j 1} / \Delta\right)_{\Delta=0},\left(g_{j 2} / \Delta\right)_{\Delta=0}(j=0,2)$ (note that $g_{11}=g_{12}=0$ because of $\left.\langle f\rangle=0\right)$. This procedure leads to $\left(\Sigma_{1}(\mathbf{k}, \omega) / \Delta\right)_{\Delta=0}=0$ and

$$
\left(\Sigma_{2}(\mathbf{k}, \omega) / \Delta\right)_{\Delta=0}=-\frac{\Gamma}{\pi N_{0}}\left[\frac{1}{c_{i}^{2}+1}\left(g_{02} / \Delta\right)_{\Delta=0}+\frac{1}{c_{a}^{2}+1}\left(g_{22} / \Delta\right)_{\Delta=0} f^{2}(\mathbf{k})\right] \operatorname{sgn}(\omega)
$$

where

$$
\begin{aligned}
& \frac{1}{\pi N_{0}}\left(g_{02} / \Delta\right)_{\Delta=0}=\left(c_{i}^{2}+1\right)\left[c_{i}^{2}+1-\Gamma\left\langle\frac{1}{\tilde{\omega}_{0}}\right\rangle\right]^{-1} \\
& \times\left[-i\left\langle\frac{e}{\tilde{\omega}_{0}}\right\rangle+\Gamma\left(c_{a}^{2}+1\right)^{-1}\left\langle\frac{f^{2}}{\tilde{\omega}_{0}}\right\rangle \frac{1}{\pi N_{0}}\left(g_{22} / \Delta\right)_{\Delta=0}\right]
\end{aligned}
$$

and

$$
\begin{aligned}
& \frac{1}{\pi N_{0}}\left(g_{22} / \Delta\right)_{\Delta=0}=-i\left(c_{a}^{2}+1\right) \\
& \times\left[\left(c_{a}^{2}+1-\Gamma\left\langle\frac{f^{4}}{\tilde{\omega}_{0}}\right\rangle\right)\left(c_{i}^{2}+1-\Gamma\left\langle\frac{1}{\tilde{\omega}_{0}}\right\rangle\right)-\Gamma^{2}\left\langle\frac{f^{2}}{\tilde{\omega}_{0}}\right\rangle^{2}\right]^{-1} \\
& \times\left[\left\langle\frac{e f^{2}}{\tilde{\omega}_{0}}\right\rangle\left(c_{i}^{2}+1-\Gamma\left\langle\frac{1}{\tilde{\omega}_{0}}\right\rangle\right)+\Gamma\left\langle\frac{f^{2}}{\tilde{\omega}_{0}}\right\rangle\left\langle\frac{e}{\tilde{\omega}_{0}}\right\rangle\right]
\end{aligned}
$$

with $\tilde{\omega}_{0}=\omega+i \Sigma_{0}(\mathbf{k}, \omega)_{\Delta=0}$. In the following subsections we consider the above self-energies in the Born and unitary scattering limits.

\section{A. Born scattering}

When both isotropic and anisotropic impurity scattering channels are in the Born scattering regime i.e. $c_{i} \gg 1\left(v_{i} N_{0} \ll 1\right), c_{a} \gg 1\left(v_{a} N_{0} \ll 1\right)$ only the lowest order terms in the impurity potential play role in the self-energies. Keeping up to the square terms in $v_{i}\left(v_{a}\right)$ we obtain from Eqs. (20) and (21)

$$
\begin{gathered}
\Sigma_{0}(\mathbf{k}, \omega)_{\Delta=0}=-i \pi n N_{0}\left[v_{i}^{2}+v_{a}^{2} f^{2}(\mathbf{k})\right] \operatorname{sgn}(\omega) \\
\Sigma_{3}(\mathbf{k}, \omega)_{\Delta=0}=n\left[v_{i}+v_{a} f^{2}(\mathbf{k})\right]
\end{gathered}
$$


Though $\Sigma_{3}$ is nonzero, it may be absorbed into the chemical potential and its effect vanishes in the Born scattering. 23 For $\left(\Sigma_{2}(\mathbf{k}, \omega) / \Delta\right)_{\Delta=0}$ we obtain

$$
\left(\Sigma_{2}(\mathbf{k}, \omega) / \Delta\right)_{\Delta=0}=i \pi n N_{0}\left[v_{i}^{2}\langle e\rangle+v_{a}^{2}\left\langle e f^{2}\right\rangle f^{2}(\mathbf{k})\right] \frac{\operatorname{sgn}(\omega)}{\omega}
$$

It is noteworthy that apart from the average value of the order parameter symmetry function, $\langle e\rangle$, a term reflecting the overlap between $e(\mathbf{k})$ and $f^{2}(\mathbf{k})$ influences the selfenergy $\left(\Sigma_{2}(\mathbf{k}, \omega) / \Delta\right)_{\Delta=0}$. The limit of $v_{a}=0$ gives the standard $s$-wave impurity scattering in the Abrikosov-Gorkov approximation 23 with $\Sigma_{0}(\mathbf{k}, \omega)_{\Delta=0}=-i \pi n N_{0} v_{i}^{2} \operatorname{sgn}(\omega)$, $\left(\Sigma_{2}(\mathbf{k}, \omega) / \Delta\right)_{\Delta=0}=i \pi n N_{0} v_{i}^{2}\langle e\rangle \operatorname{sgn}(\omega) / \omega$, and the critical temperature determined by $\ln \left(T_{c} / T_{c_{0}}\right)=\left(\langle e\rangle^{2}-1\right)\left[\Psi\left(1 / 2+n N_{0} v_{i}^{2} /\left(2 T_{c}\right)\right)-\Psi(1 / 2)\right]$.

\section{B. Unitary scattering}

The limit of the resonant impurity scattering in both isotropic and anisotropic channels i.e. $c_{i} \rightarrow 0, c_{a} \rightarrow 0$ in Eqs. (20) and (21) leads to

$$
\Sigma_{0}(\mathbf{k}, \omega)_{\Delta=0}=-i \Gamma\left[1+f^{2}(\mathbf{k})\right] \operatorname{sgn}(\omega)
$$

and $\Sigma_{3}(\mathbf{k}, \omega)_{\Delta=0}=0$ which is consistent with the assumption of particle-hole symmetry of the excitation spectrum. The unitarity limit in Eqs. (22)-(24) gives

$$
\begin{aligned}
& \left(\Sigma_{2}(\mathbf{k}, \omega) / \Delta\right)_{\Delta=0}=i \Gamma\left\{\left[1-\Gamma\left\langle\frac{1}{\tilde{\omega}_{0}}\right\rangle\right]^{-1}\left\langle\frac{e}{\tilde{\omega}_{0}}\right\rangle\right. \\
& +\left[\Gamma\left(1-\Gamma\left\langle\frac{1}{\tilde{\omega}_{0}}\right\rangle\right)^{-1}\left\langle\frac{f^{2}}{\tilde{\omega}_{0}}\right\rangle+f^{2}(\mathbf{k})\right] \\
& \times\left[\left(1-\Gamma\left\langle\frac{1}{\tilde{\omega}_{0}}\right\rangle\right)\left(1-\Gamma\left\langle\frac{f^{4}}{\tilde{\omega}_{0}}\right\rangle\right)-\Gamma^{2}\left\langle\frac{f^{2}}{\tilde{\omega}_{0}}\right\rangle^{2}\right]^{-1} \\
& \left.\times\left[\left(1-\Gamma\left\langle\frac{1}{\tilde{\omega}_{0}}\right\rangle\right)\left\langle\frac{e f^{2}}{\tilde{\omega}_{0}}\right\rangle+\Gamma\left\langle\frac{f^{2}}{\tilde{\omega}_{0}}\right\rangle\left\langle\frac{e}{\tilde{\omega}_{0}}\right\rangle\right]\right\} \operatorname{sgn}(\omega)
\end{aligned}
$$


The impurity potential may lead to the strong scattering in one channel and the weak scattering in another. If the strong scattering takes place in the isotropic channel and the scattering in the anisotropic one is in the Born limit, then we deal with a case of the isotropic unitary scattering. $33,44,40$ The opposite case with the strong scattering in the anisotropic channel and the weak scattering in the isotropic one is equivalent to a nonphysical situation of unitary scattering in the anisotropic channel alone as discussed in section II.

\section{CRITICAL TEMPERATURE}

The critical temperature in the weak-coupling BCS approximation is determined by the equation

$$
\begin{aligned}
& \ln \left(\frac{T_{c}}{T_{c_{0}}}\right)=\pi T_{c} \sum_{\omega}\left[\frac{1}{\pi N_{0}} \sum_{\mathbf{k}} e(\mathbf{k}) \frac{e(\mathbf{k})+\frac{1}{i}\left(\Sigma_{1}(\mathbf{k}, \omega) / \Delta\right)_{\Delta=0}+\frac{1}{i}\left(\Sigma_{2}(\mathbf{k}, \omega) / \Delta\right)_{\Delta=0}}{\left(\omega+i \Sigma_{0}(\mathbf{k}, \omega)_{\Delta=0}\right)^{2}+\left(\xi_{k}+\Sigma_{3}(\mathbf{k}, \omega)_{\Delta=0}\right)^{2}}\right. \\
& \left.-\frac{\operatorname{sgn}(\omega)}{\omega}\right]
\end{aligned}
$$

where because of the momentum-dependent self-energies the summations over the quasimomentum vector $\mathbf{k}$ restricted to the Fermi surface and Matsubara frequency $\omega$ need to be performed numerically. We do the calculations in two different scattering regimes - Born and unitary for the $d$-wave order parameter. Finally the results for the $(d+s)$-wave superconductor are discussed briefly. In order to proceed further one has to choose a function describing the anisotropy of the impurity potential. We study the functions given by the subsequent harmonics as they represent an orthonormal and complete set in the interval $[0$, $2 \pi$ ] and can be used for the Fourier expansion of any regular function. Harmonics up to the 4th order are analyzed and the results are extended to higher-order harmonic functions. It is important to realize that the impurity potential of $p$-wave anisotropy shows a particular property. Given by $\sin \phi$ or $\cos \phi$ function it represents a small-angle scattering which is relatively unimportant in contributing to the resistivity of the normal state. Taken even together with the $s$-wave scattering channel it results within the linear response approximation 
to the distribution function in a not well defined integrals determining the dc conductivity for some electric field orientations.28 In other words, in the Boltzmann equation based analysis of the transport properties the $p$-wave scattering amplitude couples to the " $1-\cos (\theta)$ " term in the collision integral. Therefore, in a fit to the real systems the $p$-wave scattering can be considered only as one of the components in the anisotropic channel of the impurity potential coexisting with some other higher-order harmonics. In order to emphasize this different feature of the $p$-wave potential among the other basis functions we present the momentum-dependent scattering with various amounts of the $p$-wave potential in a separate figure.

The anisotropic impurity scattering is compared to the isotropic one of the same scattering strength. It means that we discuss the effect of substituting a part of a given isotropic impurity potential $v_{0}$ with an anisotropic term. The amplitude of the replaced isotropic potential, $v_{0}$, and the amplitudes of the isotropic and anisotropic scattering channels in the studied potential are related through the formulas $v_{i}=\alpha v_{0}$, and $v_{a}=(1-\alpha) v_{0}$, where the coefficient $\alpha(0 \leq \alpha \leq 1)$ defines the partition of the potential.

\section{A. Born scattering}

The impurity scattering is analyzed as a function of the pair-breaking parameter $\Gamma^{\prime}=$ $\pi n N_{0} v_{0}^{2}\left(v_{0}=v_{i}+v_{a}\right)$ which includes the overall scattering strength that is, takes both isotropic and anisotropic channels into account. It is convenient to use the ratio $v_{a} / v_{i}=$ $(1-\alpha) / \alpha$ to define a particular potential.

\section{1. s-wave superconductor}

We note, before discussing the unconventional superconductivity, that for the isotropic s-wave superconductor, given by $e(\mathbf{k})=1$, the $T_{c}$ equation (30) along with Eqs. (25) and

(27) do not lead to a change of the critical temperature in agreement with the Anderson's theorem. 44 


\section{2. d-wave superconductor}

The critical temperature for a $d_{x^{2}-y^{2}}$-wave superconductor that is for $e(\mathbf{k}) \sim\left(k_{x}^{2}-k_{y}^{2}\right)$ is presented for a large range of $v_{a} / v_{i}$ ratio values in Fig. 1. The major common feature of these diagrams is a lower $T_{c}$ suppression by the anisotropic impurity scattering compared to the isotropic ( $s$-wave) one. According to their effect on superconductivity the anisotropic potentials can be classified in three groups defined by the function $f(\mathbf{k}): \cos 2 \phi, \sin 2 \phi$, and higher-order harmonics. The first of them, determined by $f(\mathbf{k}) \sim \cos 2 \phi$, leads to the strongest $T_{c}$ suppression. In this case the directions of the maximum impurity scattering correspond to the maxima of the order parameter and most of the pair-breaking process takes place in this region. Therefore, the suppression of superconductivity is particularly strong. However, for the level of anisotropy up to $(1-\alpha) \approx 0.5\left(v_{a} / v_{i} \approx 1\right)$ the depairing effect of impurities is reduced with increasing contribution of $\cos 2 \phi$ scattering compared to the isotropic scattering. Further increase of the anisotropic part in the impurity potential up to a level $(1-\alpha) \approx 0.6\left(v_{a} / v_{i} \approx 1.5\right)$ almost does not change the pair-breaking effect. When the contribution of the anisotropy exceeds this level the impurity effect on $T_{c}$ is enhanced and finally for $(1-\alpha)$ of the order of $0.83\left(v_{a} / v_{i}=5\right)$ becomes comparable to the one of the $s$-wave scatterers as it is shown in Fig. 1e. The out of phase scattering takes place for $f(\mathbf{k}) \sim \sin 2 \phi$. This function is a $45^{\circ}$ rotation in the xy plane of the order parameter function $e(\mathbf{k}) \sim \cos 2 \phi$, that is, the impurity potential maxima correspond to the superconducting gap nodes and vice versa. In this way the impurity pair-breaking effect is minimized. The suppression of the critical temperature is reduced by an increasing amount of the anisotropic scattering in the impurity potential. For large $v_{a} / v_{i}$, however, the shape of the suppression lines changes slightly and a small enhanced suppression can be observed for some impurity concentration (Fig. 1e). In general, the anisotropic impurity potential given by a function orthogonal to the order parameter is less pair-breaking than $f(\mathbf{k}) \sim e(\mathbf{k})$. The $T_{c}$ suppression by the 3rd and 4th order harmonics is less than the one of $\cos 2 \phi$ but it exceeds that of $\sin 2 \phi$. It is also decreasing for increasing level of anisotropy 
in the impurity potential up to $(1-\alpha) \approx 0.5$, then the pair-breaking effect of impurities practically saturates. The curvature of the graphs changes at some points, however, and the normalized critical pair-breaking parameter, $\Gamma^{\prime} /\left(2 \pi T_{c_{0}}\right)$, at which $T_{c} \approx 0$ may increase in some cases. It is worth observing that the critical temperature is almost equally suppressed by the impurity potentials given by the 3rd and 4th order harmonics for $v_{a}$ values up to $v_{a} / v_{i}=1$. This is particularly true for small anisotropic scattering levels. When the contribution of the anisotropic channel in the impurity potential is larger, the differences are more pronounced. Nevertheless, even for the amplitudes ratio as high as $v_{a} / v_{i}=2$ ( $1-\alpha \approx 0.67$ ) the curves differ only near a zero value of the critical temperature and the normalized critical pair-breaking parameters, $\Gamma^{\prime} /\left(2 \pi T_{c_{0}}\right)$, of these four harmonics are within an interval of the order of magnitude of $10^{-2}$. For the sake of transparency we present $\cos 3 \phi$ scattering effect only in Fig. 1d. Though the curves of different harmonics overlap for the most of the critical temperature range for the amplitude in the anisotropic scattering channel five times as much as the one in the isotropic channel, $v_{a} / v_{i}=5$, they split distinctly at a low temperature of about $0.2 T_{c}$ (Fig. 1e). A study of the higher order harmonics up to the 10th order for the anisotropic scattering strength equal to the isotropic one $\left(v_{a} / v_{i}=1\right)$ leads to almost the same result as the 3rd and 4th order harmonics. This approximately universal behavior for harmonics from the 3rd to the 10th order is shown in Fig. 2. Concluding we may say that to a good accuracy the anisotropy of the impurity potential given by the harmonics of the order higher than two yields an approximately universal $T_{c}$ suppression. It is also worth mentioning that except for the isotropic scattering the critical temperature goes to a zero value asymptotically. The asymptotic tails start at very low temperatures of the order of magnitude of $10^{-4} T_{c_{0}}$ (not seen at the figure scale) where the fluctuation effects become important and may destroy superconductivity.

Remembering that this potential cannot be considered on its own without any higherorder admixture, we show the pair-breaking effect of the $p$-wave scattering in the anisotropic channel for different $v_{a} / v_{i}$ values in Fig. 3. Compared to the other harmonics (Figs. 1-2) it yields the lowest $T_{c}$ suppression. This fact can be explained by an effectively small-angle 
scattering of the $p$-wave potential. If it is given by $\cos \phi$ function then mostly the quasiparticles with their momenta parallel to the $\mathrm{x}$-axis are affected, and for the sin $\phi$ function representing impurity potential the electrons moving along the y-axis are being scattered. Thus we deal with a weak practically one-dimensional scattering in a two-dimensional space. The pair-breaking effect of both basis functions $\cos \phi$ and $\sin \phi$ is the same within the accuracy of the numerical calculations. Worth observing is also a feature of a reduced $T_{c}$ suppression with an increasing amount of $p$-wave scattering in the impurity potential. However, at large amplitudes in the anisotropic channel $\left(v_{a} / v_{i} \sim 5\right)$ the initial suppression of the critical temperature can be enhanced. Characteristic is also a clear asymptotic decrease of the critical temperature to its zero value.

Any impurity potential given by Eq. (1) can be represented as a combination of the potentials based on single harmonics. Therefore, we expect that the pair-breaking effect will be given by an appropriate superposition of the effects discussed above.

\section{3. $(d+s)$-wave superconductor}

As an example of a $\left(d_{x^{2}-y^{2}}+s\right)$-wave superconductor we consider the one with an $s$-wave admixture of $10 \%$. This is the order of magnitude of the $s$-wave level which cannot be ruled out by the ARPES measurements in the cuprates. 15 , 4 The normalized to unity order parameter is given then by $e(\mathbf{k})=(\cos 2 \phi+s) /\left\langle(\cos 2 \phi+s)^{2}\right\rangle^{1 / 2}$, where $s=(0.005 / 0.99)^{1 / 2}$ and its FS average, $\langle e\rangle=0.1$, corresponds to $10 \%$ of the $s$-wave fraction in the $\left(d_{x^{2}-y^{2}}+s\right)$ wave superconductor. The results for $v_{a} / v_{i}=1$ are shown in Fig. 4. Even a small amount of the $s$-wave component, which is not destroyed by the potential scattering, results in a robustness of $T_{c}$ compared to the pure $d$-wave superconductor (Fig. 1c) and its asymptotic reduction. A change in the symmetry of the order parameter also causes a split in the suppression of the critical temperature by the $p$-wave anisotropic channel scattering. 


\section{B. Unitary scattering}

The relation determining the partition of the scattering amplitudes between the isotropic and anisotropic channels, $v_{i}+v_{a} f(\mathbf{k}) f\left(\mathbf{k}^{\prime}\right)=v_{0} \alpha+v_{0}(1-\alpha) f(\mathbf{k}) f\left(\mathbf{k}^{\prime}\right)$, holds as long as these amplitudes are finite. In the case of the resonant scattering we make an approximation of $v_{i}$ and $v_{a}$ diverging to infinity. Therefore we cannot control the relative scattering strengths in both channels and these processes become independent. Each part of the impurity potential enters the self-energy functions (Eqs. 2024) through variables $c_{i}$ and $c_{a}$. It is important to note that the self-energies depend on $c_{i}$ and $c_{a}$ parameters separately so they are functions of $c_{i}$ and $c_{a}$ and not of any combination of them (like $c_{a} / c_{i}$ for instance). In the unitarity limit $c_{i} \rightarrow 0, c_{a} \rightarrow 0$ and the contributions from the isotropic and anisotropic channels are in fact equal. Similarly to the Born scattering limit, we want to discuss the effect of an anisotropic impurity potential that replaces the isotropic one. In order to do an appropriate comparison we need to refer to the isotropic scattering in two channels or equivalently to a regular isotropic scattering in one channel with a doubled impurity concentration $2 n$. Therefore, to compare the anisotropic unitary scattering with a corresponding isotropic one, the impurity concentration for the anisotropic scattering model must be half of the concentration of the $s$-wave impurities. We take the impurity concentration equal to $n / 2$ for the two-channel anisotropic potential and the results are plotted as a function of $\Gamma^{\prime}=2 \Gamma=n /\left(\pi N_{0}\right)$, where $n$ is the real impurity concentration the same as in the Abrikosov-Gorkov scaling function for the $s$-wave scattering. That is, the two-channel scattering potential is averaged over two impurities so there is only one scattering channel per impurity present. This procedure introduces the anisotropic scattering potential of the form given by Eq. (11) and the same scattering strength as the isotropic one in the unitarity limit. 


\section{1. s-wave superconductor}

The lack of influence of the potential anisotropic impurity scattering in the unitary limit on the critical temperature can be shown rigorously for a small concentration of the defects $n /\left(\pi N_{0}\right) \ll 1$, when the self-energies $\Sigma_{0}(\mathbf{k})_{\Delta=0}=-i \Gamma\left[1+f^{2}(\mathbf{k})\right] \operatorname{sgn}(\omega)$ (Eq. (28))

and $\left(\Sigma_{2}(\mathbf{k}) / \Delta\right)_{\Delta=0}=-\Sigma_{0}(\mathbf{k})_{\Delta=0} / \omega($ Eq. (29) $)$. For a larger impurity concentration a numerical analysis confirms this result with a very good accuracy.

\section{2. d-wave superconductor}

The effect of the anisotropic unitary scattering on a $d_{x^{2}-y^{2}}$-wave superconductor is shown in Fig. 5. The same general rule as for the Born scattering holds here. The weakest pairbreaking effect is caused by the impurity potential involving $\sin 2 \phi$ and most suppression is seen for the potential containing $\cos 2 \phi$, that is the anisotropic scattering channel in phase with the order parameter. All the other harmonics orthogonal to $\cos 2 \phi$ yield a moderate suppression of the critical temperature comparable to the isotropic scattering. They also form an almost universal suppression curve. A study of the $T_{c}$ reduction by the harmonics of the order up to ten shows the same approximate universality as that in Fig. 2, with the differences in the normalized critical pair-breaking parameter, $\Gamma^{\prime} /\left(2 \pi T_{c_{0}}\right)$, of the order of $10^{-3}$. The curves of the 3rd and 4th order harmonics overlap in Fig. 5. Particularly interesting feature of the resonant scattering is a strong pair-breaking effect of the $\cos 2 \phi$ anisotropic potential. It destroys superconductivity even faster than the isotropic impurities. Another characteristic fact is practically the same critical temperature dependence on the impurity concentration (i.e. not distinguishable in Fig. 5) for the $p$-wave scattering in the anisotropic channel and $\sin 2 \phi$ scattering potential. Similarly to the weak scattering limit, except for the $s$-wave scattering the suppression of superconductivity at low $T_{c}$ is asymptotic, however not seen at the scale of Fig. 5 . 


\section{3. $(d+s)$-wave superconductor}

A $\left(d_{x^{2}-y^{2}}+s\right)$-wave superconductor is more robust against the impurity scattering due to a nonzero $s$-wave component. Since $\cos 2 \phi$ function is no longer in phase with the order parameter its pair-breaking effect is lowered and becomes even less than the isotropic one for low critical temperatures. The results for the same level of the $s$-wave part in a $\left(d_{x^{2}-y^{2}}+s\right)$ wave order parameter as the one discussed in the Born limit are shown in Fig. 6. It is worth mentioning that the $p$-wave scattering leads again to the same $T_{c}$ suppression as the $\sin 2 \phi$ anisotropic potential.

\section{COMPARISON TO EXPERIMENT}

In the overdoped samples $T_{c} / T_{c_{0}}$ data points plotted vs. impurity concentration form a universal curve independent of the critical temperature in the absence of impurities.10 40 Such a universal scaling behavior is characteristic of the impurity limited superconductivity provided the pair-breaking parameter $\Gamma^{\prime} /\left(2 \pi T_{c_{0}}\right)$ does not change with $T_{c_{0}}$. This requirement is equivalent to a constraint $N_{0} T_{c_{0}}=$ constant in the unitarity limit and may be obeyed in the overdoped systems where the critical temperature decreases with increasing hole concentration 10 There are also indications that a large residual resistivity due to Zn atoms in the cuprates corresponds to an impurity potential scattering in the unitary limit.2 Thus, we consider the case of the unitary scattering focusing on the effect of Zn dopant. Working in the Born scattering limit requires an estimation of the impurity scattering potential which can be obtained from the residual resistivity in the normal state.20,21,24,28, 30 In that analysis a clear distinction between the impurity scattering life time and the transport relaxation time, which may differ if the impurity potential is anisotropic,22 is needed. In order to compare our unitary scattering results with the experiment we have to convert the pairbreaking parameter $\Gamma^{\prime} /\left(2 \pi T_{c_{0}}\right)$ into the impurity concentration. As $\Gamma^{\prime}=n /\left(\pi N_{0}\right)$ only

the values of the density of states on the Fermi surface and the critical temperature in the 
absence of impurities are needed for that purpose. The density of states at the Fermi level is estimated from the measurements of the specific heat jump at the phase transition $\Delta C$. We employ the BCS weak-coupling relation $\Delta C / \gamma T_{c} \approx 1.43$ to obtain the normal-state Sommerfeld constant $\gamma$ which gives the density of states through $\gamma=2 \pi^{2} k_{B}^{2} N_{0} / 3$. It is important to note that the strong-coupling corrections $\$ 9,50$ as well as the interaction with impuritie\$51,52 may change this relation significantly and in consequence alter the overall density of states. It may result in a wide range of $T_{c}$ solutions.53 Because of a difficulty in the separation of lattice and electron contributions to the specific heat the thermodynamic experiments provide $\Delta C$ values with the accuracy depending on the quality of a sample. For high purity, fully oxygenated $\mathrm{Y}-123$ compound grown in $\mathrm{BaZrO}_{3}$ the mean-field component of the electronic specific heat jump is estimated as $56 \pm 2\left(\mathrm{~mJ} / \mathrm{K}^{2}\right.$ mole $) .4855$ In La-214 system this quantity is in the range of $14 \pm 5\left(\mathrm{~mJ} / \mathrm{K}^{2}\right.$ mole $) .6$. 6 . There is no specific heat jump observed at the phase transition in $B i-2122$ compound 48 thus the present method of obtaining the density of states cannot be applied in this case. We discuss the experimental results in this system together with $Y-123$ and $L a-214$ compounds more extensively in Ref. 53. The density of states calculated from the above $\Delta C$ values are used for the evaluation of the impurity concentration, which is represented as the per cent number of impurities (defects) per planar $\mathrm{Cu}$ site. We do the calculations with a fixed density of states as we do not have any quantitative data showing its change with doping or disorder. Therefore, the results are not really universal and depend on the critical temperature in the absence of impurities $T_{c_{0}}$. We group them according to the values of the critical temperature of a pure system. In the optimally doped $Y-123$ compound $T_{c_{0}}$ varies in a very narrow range of values and for the theoretical calculation we take $T_{c_{0}}=91 \mathrm{~K}$. The phase diagrams for the $d$-wave superconductor together with the experimental data of $\mathrm{Zn}$ doped samples $13-17$ are shown in Fig. 7. The scattering effect of a given potential corresponds to the area between two curves of the same style, for instance the solid lines stand for the $\sin 2 \phi$ impurity potential. This broadened range of values stem from the uncertainty in the estimation of the FS density of states, that is, from the accuracy of the $\Delta C$ values. We note, that 
all the experimental points fall in the region of the theoretically predicted $T_{c}$ suppression by the unitary impurity scattering. Interestingly, the pair-breaking effect of Zn atoms in certain samples corresponds to the resonant scattering in different anisotropic channel of the impurity potential. We can clearly distinguish data that can be approximated by the scattering potentials given by $\cos 2 \phi$ and $\sin 2 \phi$ functions. The rest of the experimental points lie in the range of isotropic scattering or anisotropic scattering with higher order harmonics. Although the critical temperatures of the analyzed samples are very close, we do not see a universal suppression dependence as in Bi-2122 and $L a-214$ compounds.10 47 This feature may suggest a possible sample-dependence of the effective impurity scattering caused for instance by the differences in a sample preparation or Zn substitution processes. Even small changes in the hole concentration alter the electronic density of states at the Fermi level and result in a modified impurity scattering rate.

According to $T_{c_{0}}$ values, we gather the experimental data for Zn doped $L a-214$ in two groups corresponding to $T_{c_{0}}$ equal to $30 K$ (Fig. 8a) li $^{\text {and }} 36 K$ (Fig. 8b) 国目 For the sample of $T_{c_{0}} \approx 30 \mathrm{~K}$ the experimental suppression of the critical temperature is in the range of anisotropic scattering determined by $\sin 2 \phi$ (Fig. 8a). The pair-breaking effect of Zn atoms in the samples characterized by $T_{c_{0}} \approx 36 \mathrm{~K}$ is on the edge of anisotropic scattering in higher order harmonics but also right in the middle of $\sin 2 \phi$ based anisotropic scattering. As we can see, the quantitative calculations for $L a-214$ compound contain a large uncertainty margin which is caused by a lack of a precise value of the specific heat jump at the phase transition and consequently of the electron density of states on the Fermi surface. The error in the experimentally estimated magnitude of $\Delta C$ is of the order of $36 \%$.

A similar analysis of $\mathrm{Ni}$ substituted samples shows that the pair-breaking effect in the unitary limit is stronger than observed experimentally. For the sake of comparison we present Ni doped $Y-123$ compound data 12, 14, 15, 18 and our theoretical curves for the resonant scattering in Fig. 9. Less difference between the experimental and theoretical results is seen for $L a-21453$ Although this comparison is suggestive for a weak potential scattering, the detailed calculations in the Born scattering limit giving the critical temperature dependence 
on the residual resistivity 28 are needed in order to draw more firm conclusions.

Finally we discuss the electron irradiation experiments in $Y-123.19-22$ The results for low-energy (60-120 keV) incident electrons read from Fig. 12 of Ref. 21 are shown in Fig. 10. The experimental points are in the range of the pair-breaking effect of the scattering potential with the anisotropy given by $\sin 2 \phi$. The initial $T_{c}$ suppression, however, is more gradual than the one obtained from the theoretical calculation and cannot be explained by the standard impurity pair-breaking mechanism. Unfortunately, we do not have any other set of electron irradiation data representing the change of the critical temperature with defect concentration. The analysis by Tolpygo et. al.21 shows that the initial $T_{c}$ suppression for low-energy $(100 \mathrm{keV})$ electron irradiation reported by Legris et al.22 agrees with the one shown in Fig. 10. For higher-energy electrons 19 as in Ref. 21 which becomes in the range of the $\sin 2 \phi$ determined behavior for low defect concentration.

\section{CONCLUSIONS}

We have studied the pair-breaking effect of the anisotropic impurity scattering in the t-matrix approximation for $d_{x^{2}-y^{2}}$-wave and $\left(d_{x^{2}-y^{2}}+s\right)$-wave superconductors. The Born and the unitary limits have been discussed analytically and numerically. Although limited to the employed phenomenological model the conclusions we draw should be suggestive to a larger class of potentials capturing the feature of anisotropy.

We have shown, that the effect of the anisotropic impurity scattering can be considered in four groups for the Born scattering and three groups in the case of unitary scattering which are determined by the form of the function $f(\mathbf{k})$ defining symmetry of the scattering potential. In both scattering limits the strongest suppression of the critical temperature is caused by the impurity potential given by $f(\mathbf{k}) \sim \cos 2 \phi$ which is in phase with the order parameter for the $d_{x^{2}-y^{2}}$ state and overlaps significantly with the $\left(d_{x^{2}-y^{2}}+s\right)$-wave state of a major $d$-wave component. The pair-breaking effect of this potential with a large 
amount of anisotropic amplitude can be comparable with the one of the isotropic scattering in the Born limit and exceeds the $s$-wave impurity effect in the unitary limit. This issue is particularly important as it shows that a weak reduction of $T_{c}$ due to anisotropy of the impurity potential proportional to the superconducting order parameter which follows from the weak-scattering model 30 is not a general feature of the anisotropic impurity scattering. Another class is defined by $f(\mathbf{k}) \sim \sin 2 \phi$. It leads to the lowest impurity pair-breaking effect in the case of the unitary scattering and second lowest for the Born limit. This kind of scattering is maximal in the direction of the nodes of the order parameter and it vanishes where the gap function has its maxima. Therefore, the effective scattering is minimized by the symmetry of the impurity potential. Any other function orthogonal to the $d$-wave order parameter results in a rather universal $T_{c}$ suppression and falls into the third group of the potentials. The pair-breaking in this case is less than that of the isotropic scattering but it exceeds the one of $\sin 2 \phi$ based potential, and in the unitary limit is very close to the isotropic scattering. Resonant scatterers in the $p$-wave channel lead to the same $T_{c}$ suppression as the $d$-wave scattering given by $\sin 2 \phi$ function. In the Born scattering limit, however, the $p$-wave anisotropic scattering results in the lowest $T_{c}$ suppression.

We have compared our results for the $d_{x^{2}-y^{2}}$-wave superconductor with the experimental data assuming that the impurity potential is close to the unitary limit. Within the accuracy of our calculations the $\mathrm{Zn}$ atoms can be considered as the resonant scatterers in overdoped $L a-214$ and $Y-123$ compounds. The pair-breaking effect of the structure defects produced by the electron irradiation is also in the range of magnitude of the unitary scattering with the anisotropy of the impurity potential given by $\sin 2 \phi$ function. The theoretical results strongly depend on the value of the electronic density of states on the Fermi surface. Thus far, for some systems (like $L a-214$ ) there is only an estimation of this value with a large margin of uncertainty available. It opens a wide range of possible $T_{c}$ solutions and makes a proper analysis very difficult. Therefore, before any final conclusion about the impurity suppression of the critical temperature in the cuprates can be made, a much more accurate determination of the electronic density of states on the Fermi surface has to be done. 


\section{ACKNOWLEDGMENTS}

We would like to thank B. Nachumi for his help in analyzing the experimental data, as well as T. Kluge for the discussions on the effect of impurities in high- $T_{c}$ superconductors and pointing to us several important papers. We are also grateful to A. Junod for providing us with the specific heat data and for the enlightening explanations of the thermodynamic measurements in the cuprates. Finally, we would like to thank P. Hirschfeld for critical comments on the manuscript.

This work was supported by the Natural Sciences and Engineering Research Council of Canada. 


\section{REFERENCES}

* present address: Institute of Physics, Politechnika Wrocławska, Wybrzeże Wyspiańskiego 27, 50-370 Wrocław, Poland

${ }^{1}$ B. Nachumi, A. Keren, K. Kojima, M. Larkin, G. M. Luke, J. Merrin, O. Tchernyshöv, Y. J. Uemura, N. Ichikawa, M. Goto, and S. Uchida, Phys. Rev. Lett. 77, 5421 (1996)

${ }^{2}$ Y. Fukuzumi, K. Mizuhashi, K. Takenaka, and S. Uchida, Phys. Rev. Lett. 76, 684 (1996)

${ }^{3}$ G. Xiao, M. Z. Cieplak, J. Q. Xiao, and C. L. Chien, Phys. Rev. B42, 8752 (1990)

${ }^{4}$ H. Fujishita and M. Sato, Solid State Commun. 72, 529 (1989)

${ }^{5}$ R. Yoshizaki, N. Ishikawa, H. Sawada, E. Kita, and A. Tasaki, Physica C 166, 417 (1990)

${ }^{6}$ A. Maeda, T. Yabe, S. Takebayashi, M. Hase, and K. Uchinokura, Phys. Rev. B41, 4112 (1990)

${ }^{7}$ K. Westerholt and B. vom Hedt, J. Low. Temp. Phys. 95, 123 (1994)

${ }^{8}$ B. Lönnberg, T. Lundström, and P. Norling, Physica C 191, 147 (1992)

${ }^{9}$ P. S. Prabhu, M. S. R. Rao, and G. V. S. Rao, Physica C 211, 279 (1993)

${ }^{10}$ T. Kluge, Y. Koike, A. Fujiwara, M. Kato, T. Noji, and Y. Saito, Phys. Rev. B52, 727 (1995)

${ }^{11}$ T. Kluge, A. Fujiwara, M. Kato, and Y. Koike, Phys. Rev. B54, 86 (1996)

12 M. Speckmann, Th. Kluge, C. Tomé-Rosa, Th. Becherer, and H. Adrian, Phys. Rev. B47, $15185(1993)$

13 T. R. Chien, Z. Z. Wang, and N. P. Ong, Phys. Rev. Lett. 67, 2088 (1991)

${ }^{14}$ Y. Zhao, H. K. Liu, G. Yang, and S. X. Dou, J. Phys.: Condens. Matter 5, 3623 (1993)

${ }^{15}$ R. Liang, T. Nakamura, H. Kawaji, M. Itoh, and T. Nakamura, Physica C 170, 307 (1990) 
${ }^{16}$ H. Harashina, T. Nishikawa, T. Kiyokura, S. Shamoto, M. Sato, and K. Kakurai, Physica C 212, $142(1993)$

${ }^{17}$ B. Jayaram, S. K. Agarwal, C. V. N. Rao, and A. V. Narlikar, Phys. Rev. B38, 2903 (1988)

18 J. -T. Kim, D. G. Xenikos, A. Thorns, and T. R. Lemberger, J. Appl. Phys. 72, 803 (1992)

${ }^{19}$ J. Giapintzakis, D. M. Ginsberg, M. A. Kirk and S. Ockers, Phys. Rev. B 50, 15967 (1994)

${ }^{20}$ S. Tolpygo, J. -Y. Lin, M. Gurvitch, S. Y. Hou, and J. M. Phillips, Phys. Rev. B53, 12454 (1996)

${ }^{21}$ S. Tolpygo, J. -Y. Lin, M. Gurvitch, S. Y. Hou, and J. M. Phillips, Phys. Rev. B53, 12462 (1996)

${ }^{22}$ A. Legris, F. Rullier-Albenque, E. Radeva, and P. Lejay, J. Phys. I France 3, 1605 (1993)

${ }^{23}$ A. A. Abrikosov and L. P. Gorkov, Zh. Eksp. Teor. Fiz. 39, 1781 (1960) [Sov. Phys. JETP 12, 1243 (1961)]; see also A. A. Abrikosov, L. P. Gorkov,and I. E. Dzyaloshinski, Methods of Quantum Field Theory in Statistical Physics (Dover, New York, 1975), sec 39

${ }^{24}$ R. J. Radtke, K. Levin, H.-B. Schüttler and M. R. Norman, Phys. Rev. B48, 653 (1993)

${ }^{25}$ A. A. Abrikosov, Physica C 214, 107 (1993)

26 T. Hotta, J. Phys. Soc. Jpn. 62, 274 (1993)

${ }^{27}$ A small inclusion of the $s$-wave component does not change this result significantly as it has been shown in H. Kim and E. J. Nicol, Phys. Rev. B52, 13576 (1995), and M. T. Béal-Monod and K. Maki, Phys. Rev. B53, 5775 (1996)

${ }^{28}$ G. Harań and A. D. S. Nagi, in preparation

${ }^{29}$ A. J. Millis, S. Sachdev and C. M. Varma, Phys. Rev. B37, 4975 (1988)

${ }^{30}$ G. Harań and A. D. S. Nagi, Phys. Rev. B54, 15463 (1996) 
${ }^{31}$ The same result has been also obtained in M. L. Kulić and V. Oudovenko, Solid State Commun. 104, 375 (1997)

${ }^{32}$ C. H. Choi, Phys. Rev. B52, 16199 (1995)

${ }^{33}$ H. Shiba, Prog. Theor. Phys. 40, 435 (1968)

${ }^{34}$ A. I. Rusinov, Zh. Eksp. Teor. Fiz. 56, 2047 (1996) [Sov. Phys. JETP 29, 1101 (1969)]

${ }^{35}$ S. Haas, A. V. Balatsky, M. Sigrist, and T. M. Rice, Phys. Rev. B56, 5108 (1997)

${ }^{36}$ T. Kluge, G. Jakob, M. Speckmann, C. Tomé-Rosa, and H. Adrian, J. Low Temp. Phys. 105, $1415(1996)$

${ }^{37}$ J. M. Ziman, Electrons and Phonons (Oxford, London, 1963)

${ }^{38}$ It should be noted that the potential discussed in Refs. 33-35 does not have this property and is virtually isotropic unless the cylindrical FS symmetry is broken.

${ }^{39}$ G. D. Mahan, Many-Particle Physics (Plenum, New York, 1981)

${ }^{40}$ P. J. Hirschfeld, D. Vollhardt and P. Wölfle, Solid State Commun. 59, 111 (1986)

${ }^{41}$ P. J. Hirschfeld, P. Wölfle and D. Einzel, Phys. Rev. B 37, 83 (1988)

${ }^{42}$ J. Keller, K. Scharnberg and H. Monien, Physica C 152, 302 (1988)

${ }^{43}$ R. Fehrenbacher, Phys. Rev. Lett. 77, 1849 (1996)

${ }^{44}$ P. W. Anderson, J. Phys. Chem. Solids 11, 26 (1959)

${ }^{45}$ Z. X. Shen et al., Phys. Rev. Lett. 70, 1553 (1993); T. Yokoya, T. Takahashi, T. Mochiku, and K. Kadowaki, Phys. Rev. B53, 14055 (1996)

${ }^{46}$ H. Ding et al., Phys. Rev. Lett. 74, 2784 (1995); M. R. Norman, M. Randeria, H. Ding, J. C. Campuzano and A. F. Bellman, Phys. Rev. B52, 15107 (1995)

${ }^{47} \mathrm{~T}$. Kluge, private communication 
${ }^{48}$ A. Junod, private communication

${ }^{49}$ F. Marsiglio, R. Akis, and J. P. Carbotte, Phys. Rev. B36, 5245 (1987)

${ }^{50}$ J. Blezius and J. P. Carbotte, Phys. Rev. B36, 3622 (1987)

${ }^{51}$ G. Harań, J. Taylor, and A. D. S. Nagi, Phys. Rev. B55, 11778 (1997)

${ }^{52}$ G. Harań, J. Taylor, and A. D. S. Nagi, Physica C 277, 70 (1997)

${ }^{53}$ G. Harań and A. D. S. Nagi, preprint

${ }^{54}$ A. Junod, M. Roulin, J. -Y. Genoud, B. Revaz, A. Erb, E. Walker, Physica C 275, 245 (1997)

${ }^{55}$ A. Junod, M. Roulin, B. Revaz, A. Mirmelstein, J. -Y. Genoud, E. Walker, and A. Erb, Physica C 282-287, 1399 (1997)

${ }^{56}$ A. Junod, in Physical Properties of High Temperature Superconductors II, ed. D. M. Ginsberg (World Scientific, Singapore 1990), p. 13 


\section{FIGURE CAPTIONS}

Fig. 1. Normalized critical temperature $T_{c} / T_{c_{0}}$ of the $d_{x^{2}-y^{2}}$-wave superconductor as a function of the normalized impurity scattering rate $\Gamma^{\prime} /\left(2 \pi T_{c_{0}}\right)$ in the Born limit for the impurity potential symmetry (solid curves from the top): $\sin 2 \phi, \sin 3 \phi$ and higher-order harmonics, $\cos 2 \phi$. The amount of anisotropy in the potential is: (a) $(1-\alpha) \approx 0.17\left(v_{a} / v_{i}=0.2\right)$, (b) $(1-\alpha) \approx 0.33\left(v_{a} / v_{i}=0.5\right),(\mathrm{c})(1-\alpha)=0.5\left(v_{a} / v_{i}=1.0\right),(\mathrm{d})(1-\alpha) \approx 0.67$ $\left(v_{a} / v_{i}=2.0\right),(\mathrm{e})(1-\alpha) \approx 0.83\left(v_{a} / v_{i}=5.0\right)$ the higher-order harmonics split in this case into (from the top): $\sin 4 \phi, \cos 3 \phi, \sin 3 \phi, \cos 4 \phi$. The dashed line represents isotropic suppression of the critical temperature.

Fig. 2. Shaded area represents the effect of the anisotropic impurity potentials given by cosl $\phi$ and $\operatorname{sinl} \phi$ functions for $3 \leq l \leq 10$ and $(1-\alpha)=0.5\left(v_{a} / v_{i}=1.0\right)$ in the $d_{x^{2}-y^{2} \text {-wave }}$ state in the Born limit.

Fig. 3. The $d_{x^{2}-y^{2}}$-wave state $T_{c}$ suppression by the Born scattering $p$-wave impurity potential with $f(\mathbf{k})=\sin \phi($ or $\cos \phi)$ and the anisotropic scattering strength $(1-\alpha)\left(v_{a} / v_{i}\right)$ equal to (from the top) $0.83(5.0), 0.67(2.0), 0.50(1.0), 0.33(0.5), 0.17(0.2)$. The isotropic scattering is shown with the dashed line.

Fig. 4. Normalized critical temperature $T_{c} / T_{c_{0}}$ for the $\left(d_{x^{2}-y^{2}}+s\right)$-wave superconductor $(\langle e\rangle=0.1)$ as a function of the normalized impurity scattering rate $\Gamma^{\prime} /\left(2 \pi T_{c_{0}}\right)$ in the Born limit for the impurity potential symmetry (solid curves from the top): $\cos \phi, \sin \phi, \sin 2 \phi$, $\sin 3 \phi$ and higher-order harmonics, $\cos 2 \phi$. The amount of anisotropy in the potential is $(1-\alpha)=0.5\left(v_{a} / v_{i}=1.0\right)$. The dashed curve represents the effect of the isotropic impurity scattering.

Fig. 5. Normalized critical temperature $T_{c} / T_{c_{0}}$ of the $d_{x^{2}-y^{2}}$-wave superconductor as a 
function of the normalized impurity scattering rate $\Gamma^{\prime} /\left(2 \pi T_{c_{0}}\right)$ in the unitary limit for the impurity potential symmetry (solid curves from the top): $\sin 2 \phi$ (and $p$-wave), $\sin 3 \phi$ and higher-order harmonics, $\cos 2 \phi$. The dashed curve represents the effect of the isotropic impurity scattering.

Fig. 6. Normalized critical temperature $T_{c} / T_{c_{0}}$ of the $\left(d_{x^{2}-y^{2}}+s\right)$-wave superconductor $(\langle e\rangle=0.1)$ as a function of the normalized impurity scattering rate $\Gamma^{\prime} /\left(2 \pi T_{c_{0}}\right)$ in the unitary limit for the impurity potential symmetry (solid curves from the top): $\sin 2 \phi$ (and $p$-wave), $\sin 3 \phi$ and higher-order harmonics, $\cos 2 \phi$. The dashed curve represents the effect of the isotropic impurity scattering.

Fig. 7. Critical temperature of Zn substituted optimally doped $Y-123$ samples vs impurity concentration per cent per planar $\mathrm{Cu}$ site, $n_{Z n}$. The data are taken from Refs. 13 (filled circles), 14 (open squares), 15 (diamonds), 16 (open circles), 17 (filled squares). The area between the curves of the same sort corresponds to the pair-breaking effect of the unitary scattering impurities of the following anisotropies: $\sin 2 \phi$ (solid), $\cos 3 \phi$ and higher-order (long dashed), $\cos 2 \phi$ (dashed). The isotropic impurity scattering is represented by the dotdashed curves.

Fig. 8. Critical temperature of Zn substituted $L a-214$ samples vs impurity concentration per cent per planar $\mathrm{Cu}$ site, $n_{Z n}$. The data are taken from Refs. 1 (filled squares), 3 (open squares). The theoretical curves in the unitary limit are drawn for two critical temperatures: (a) $T_{c_{0}}=30 K$, (b) $T_{c_{0}}=36 K$. The impurity potentials represented by different curves agree with the notation in Fig. 7.

Fig. 9. Critical temperature of Ni substituted optimally doped $Y-123$ samples vs impurity concentration per cent per planar $\mathrm{Cu}$ site, $n_{N i}$. The data are taken from Refs. 12 (open circles), 14 (filled squares), 15 (diamonds), 18 (open squares). The theoretically calculated 
unitary scattering curves correspond to the potentials described as in Fig. 7.

Fig. 10. Critical temperature of optimally doped $Y-123$ sample with in-plane oxygen vacancies induced by $60-120 \mathrm{keV}$ electron irradiation (Ref. 21) vs defect concentration per cent per planar $\mathrm{Cu}$ site, $n_{(O \text { defects })}$. The theoretically calculated unitary scattering curves correspond to the potentials described as in Fig. 7. 


\section{FIGURES}

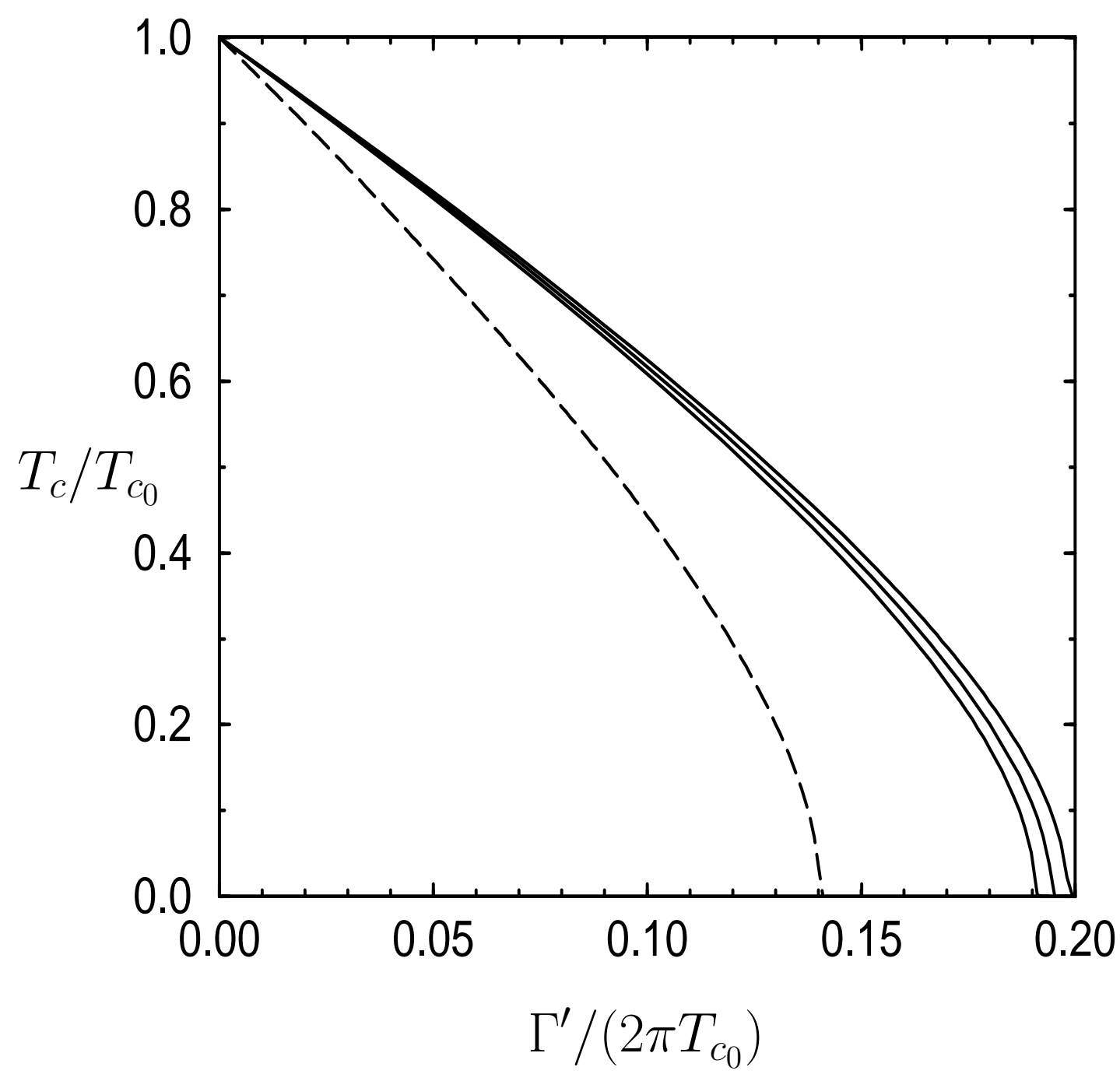

FIG. 1. Fig.1a: 


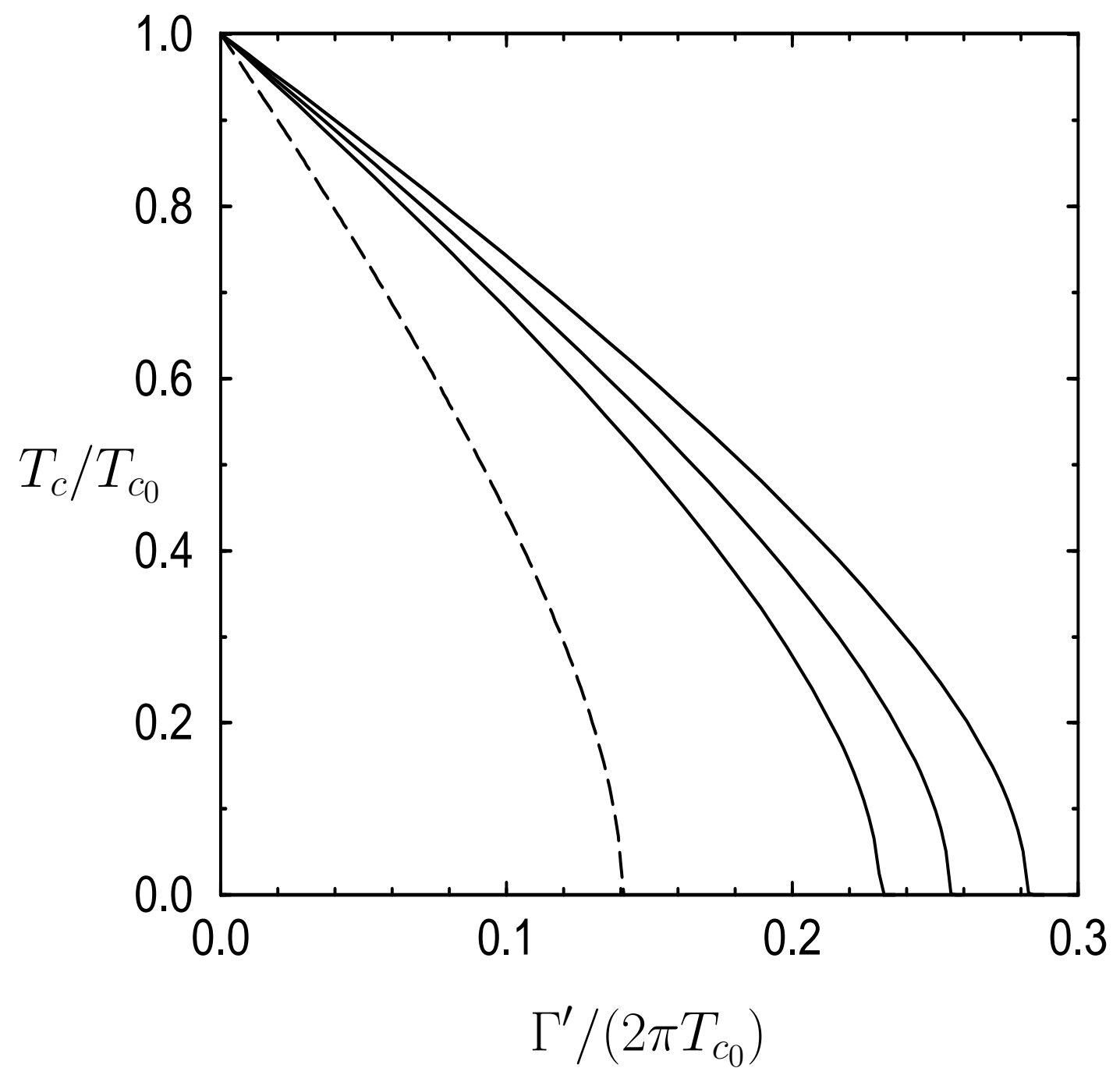

FIG. 2. Fig.1b: 


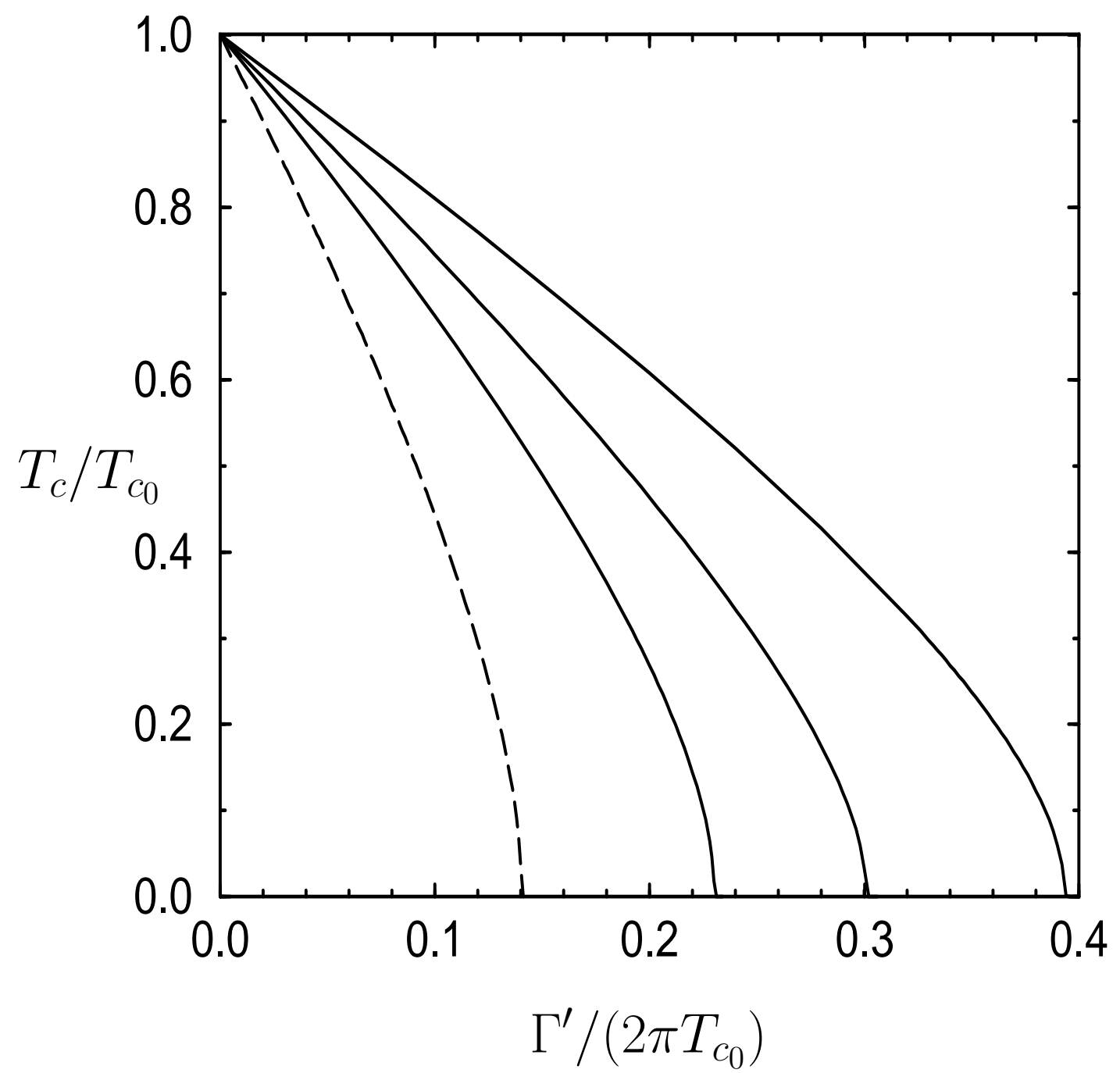

FIG. 3. Fig.1c: 


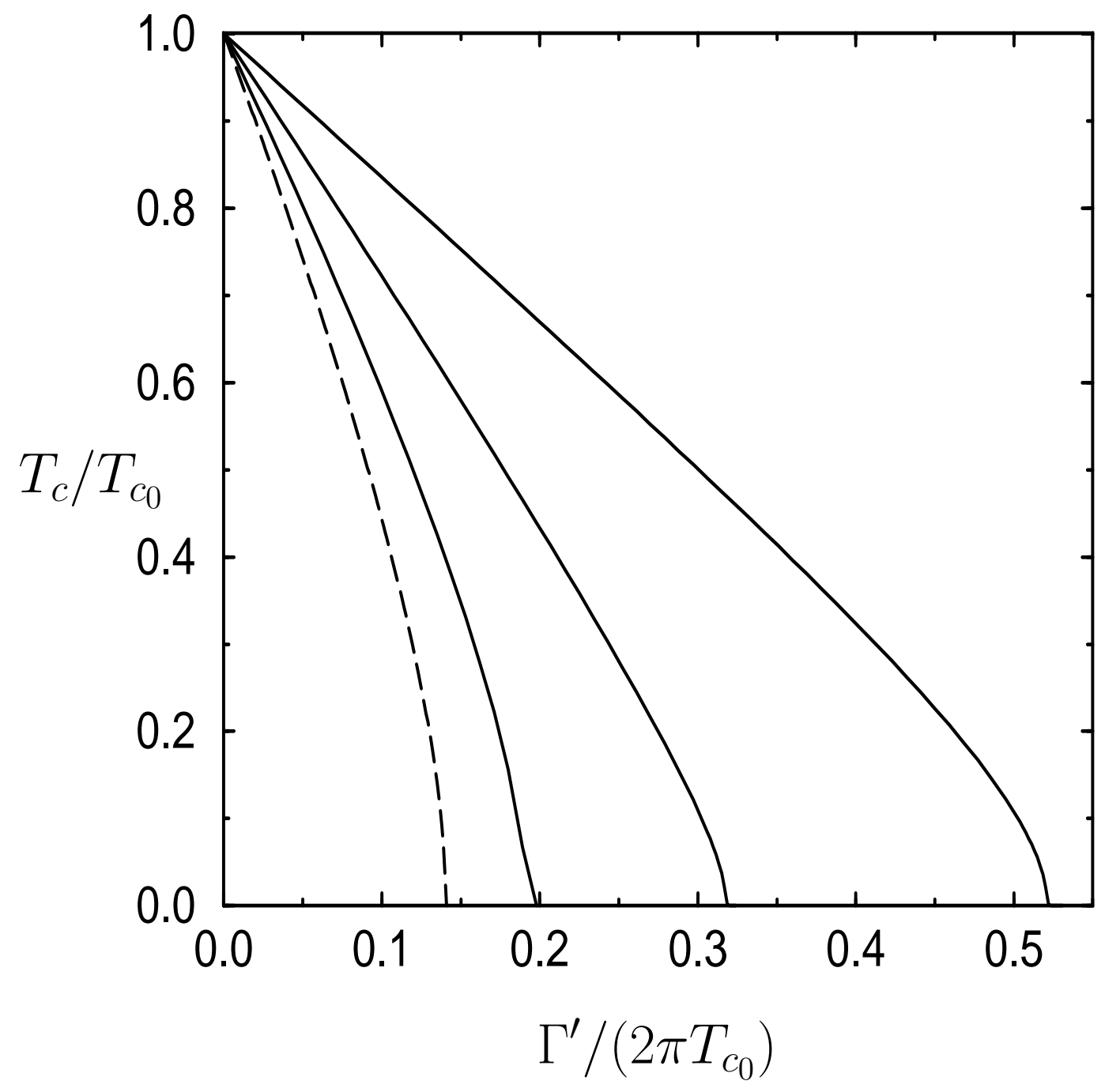

FIG. 4. Fig.1d: 


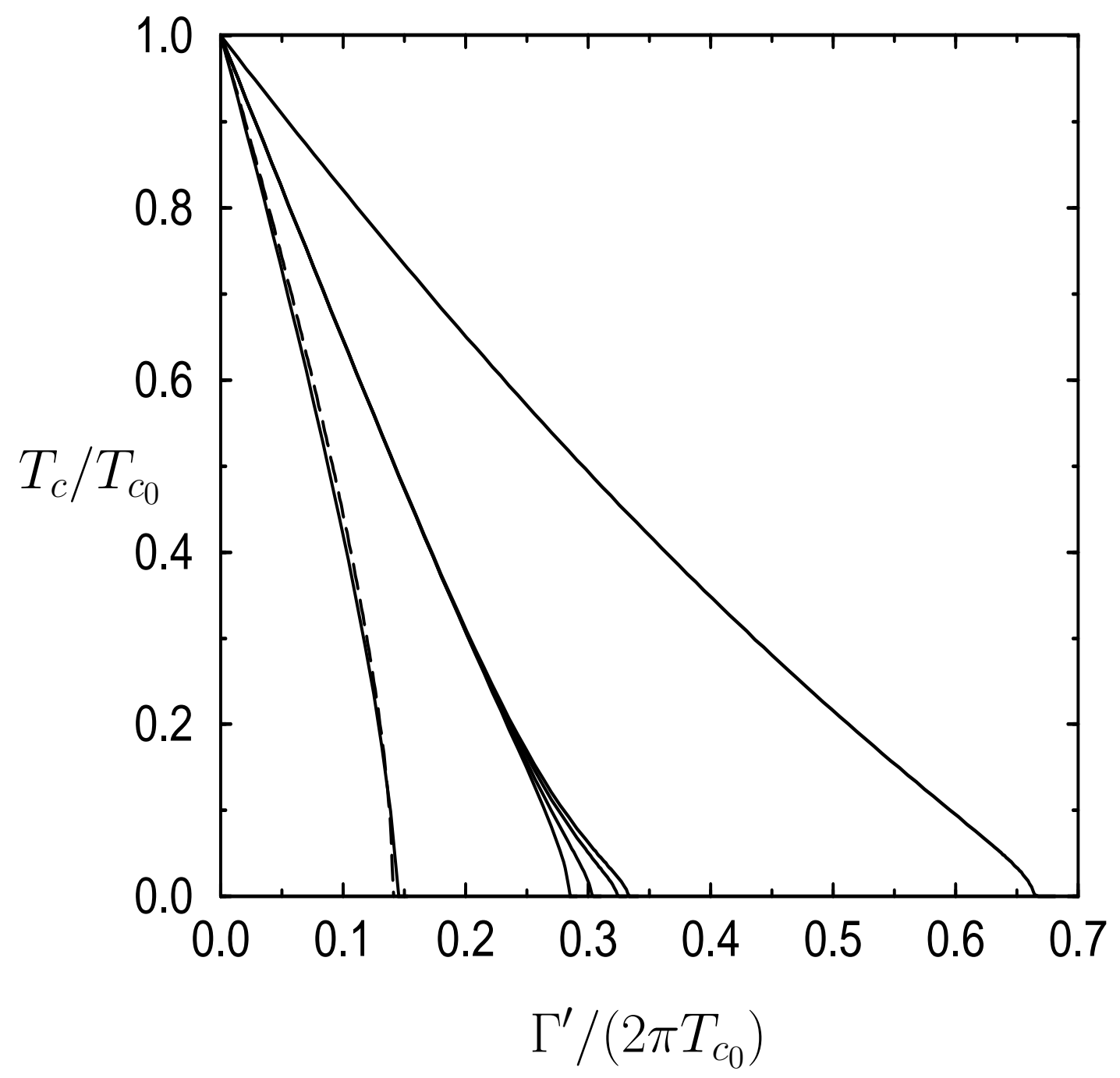

FIG. 5. Fig.1e: 


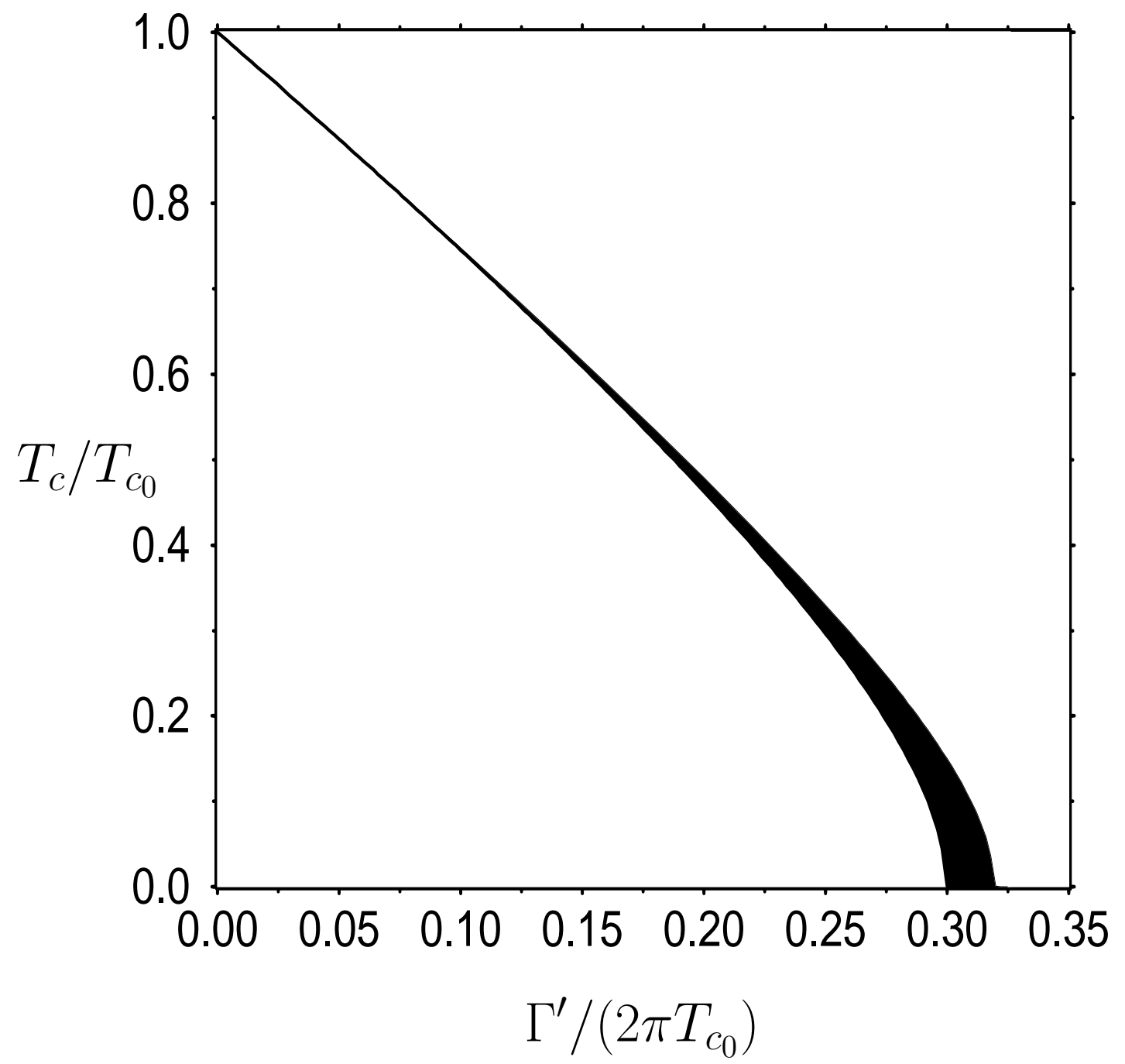

FIG. 6. Fig.2: 


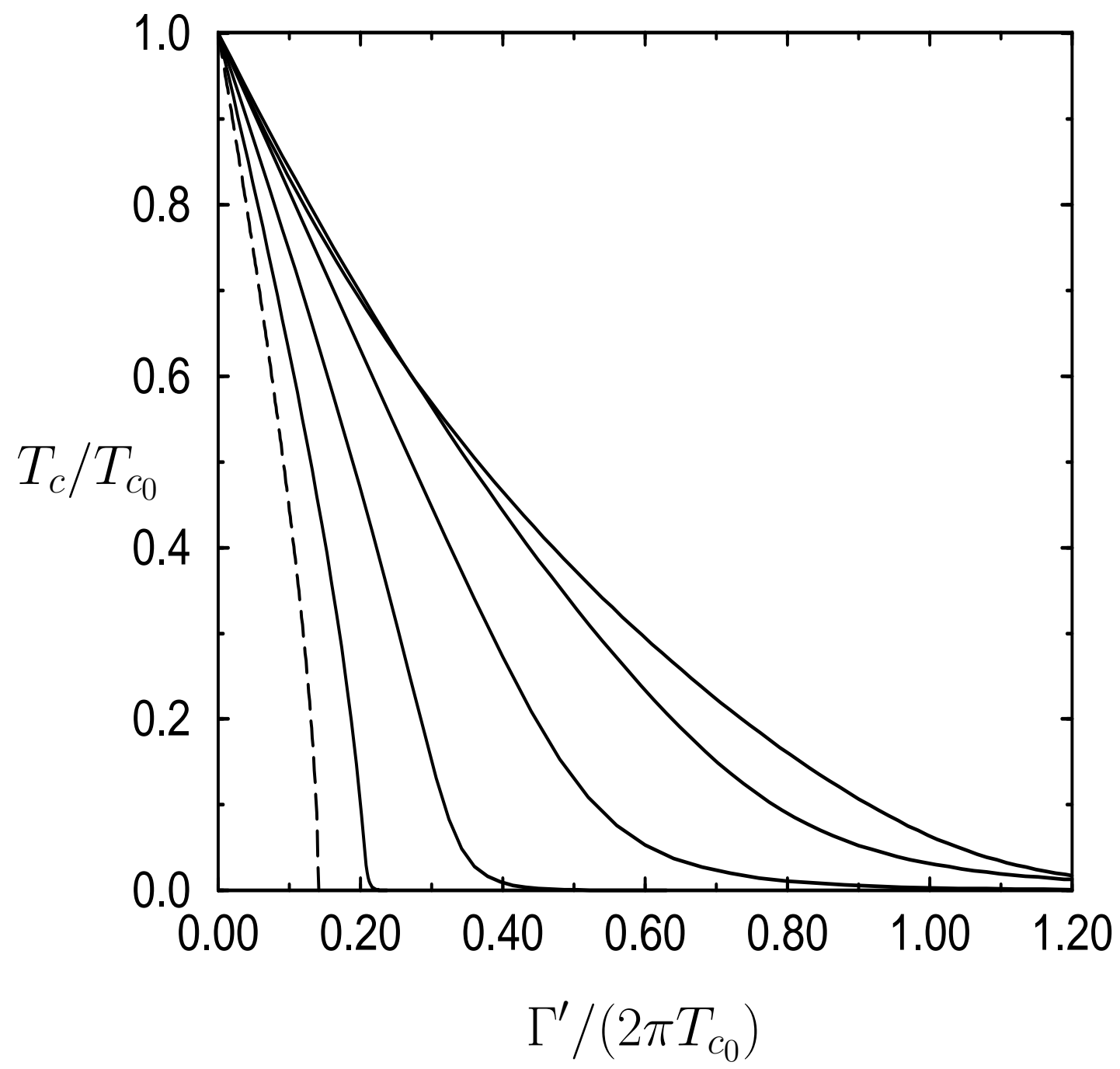

FIG. 7. Fig.3: 


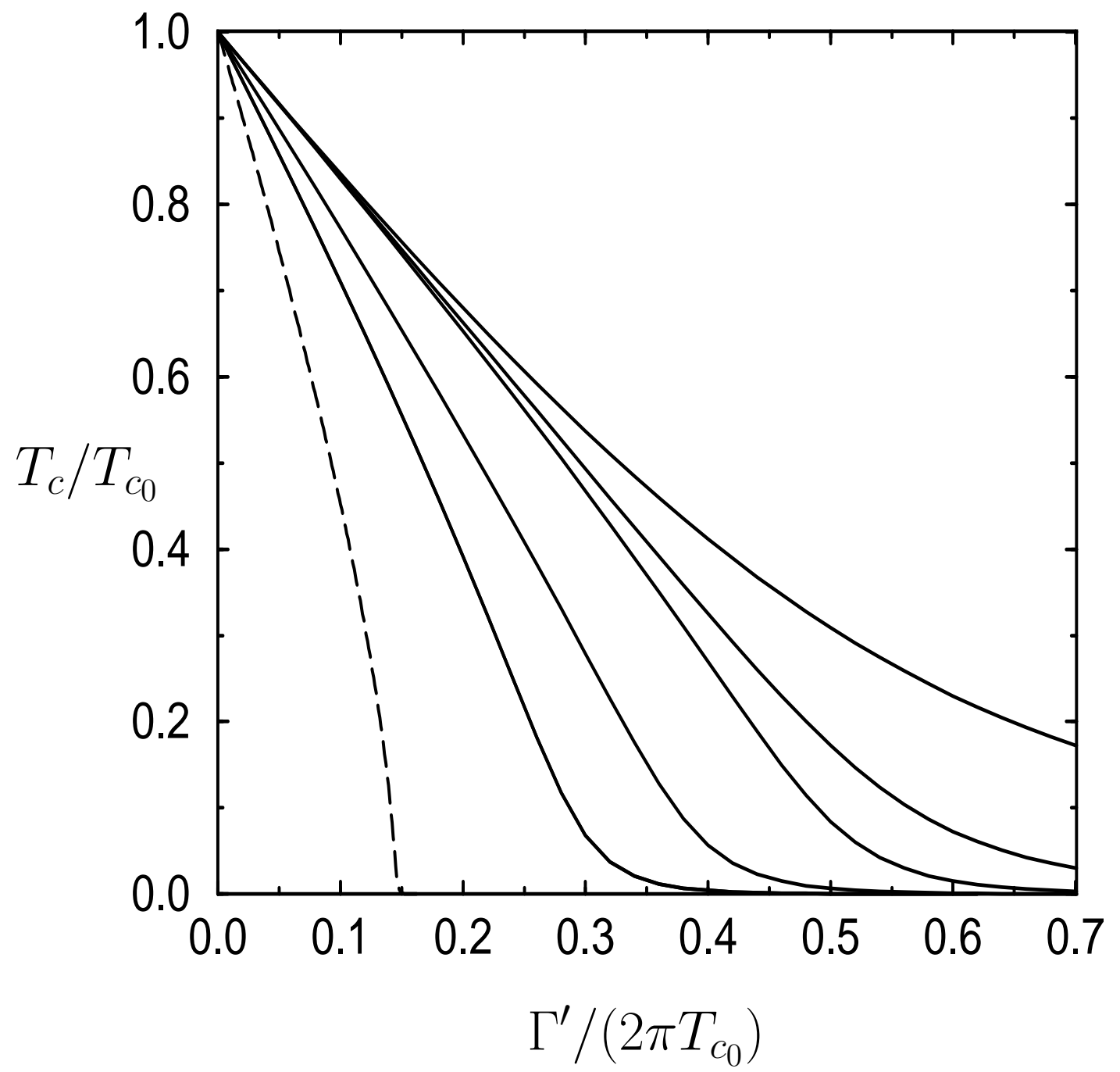

FIG. 8. Fig.4: 


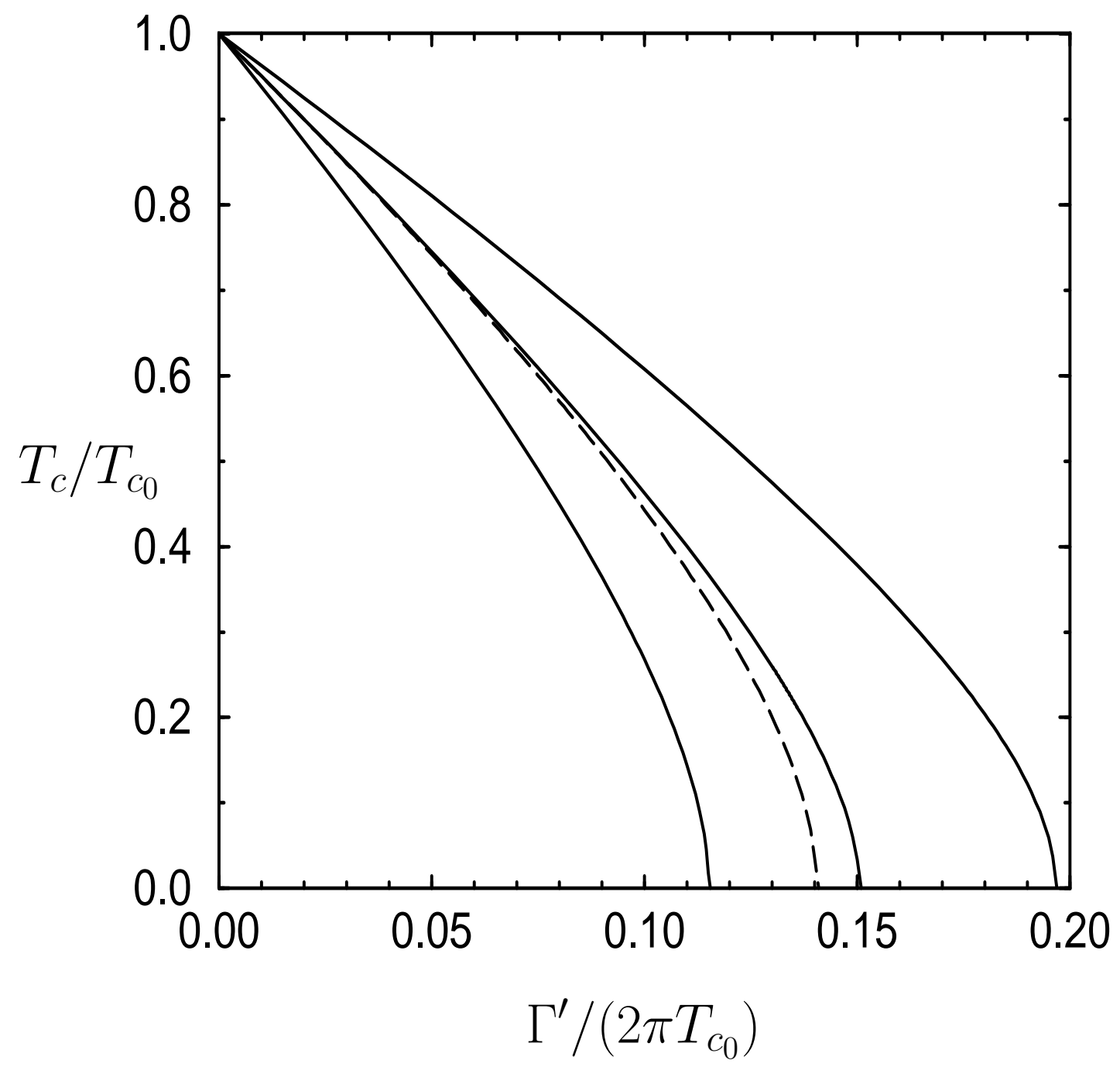

FIG. 9. Fig.5: 


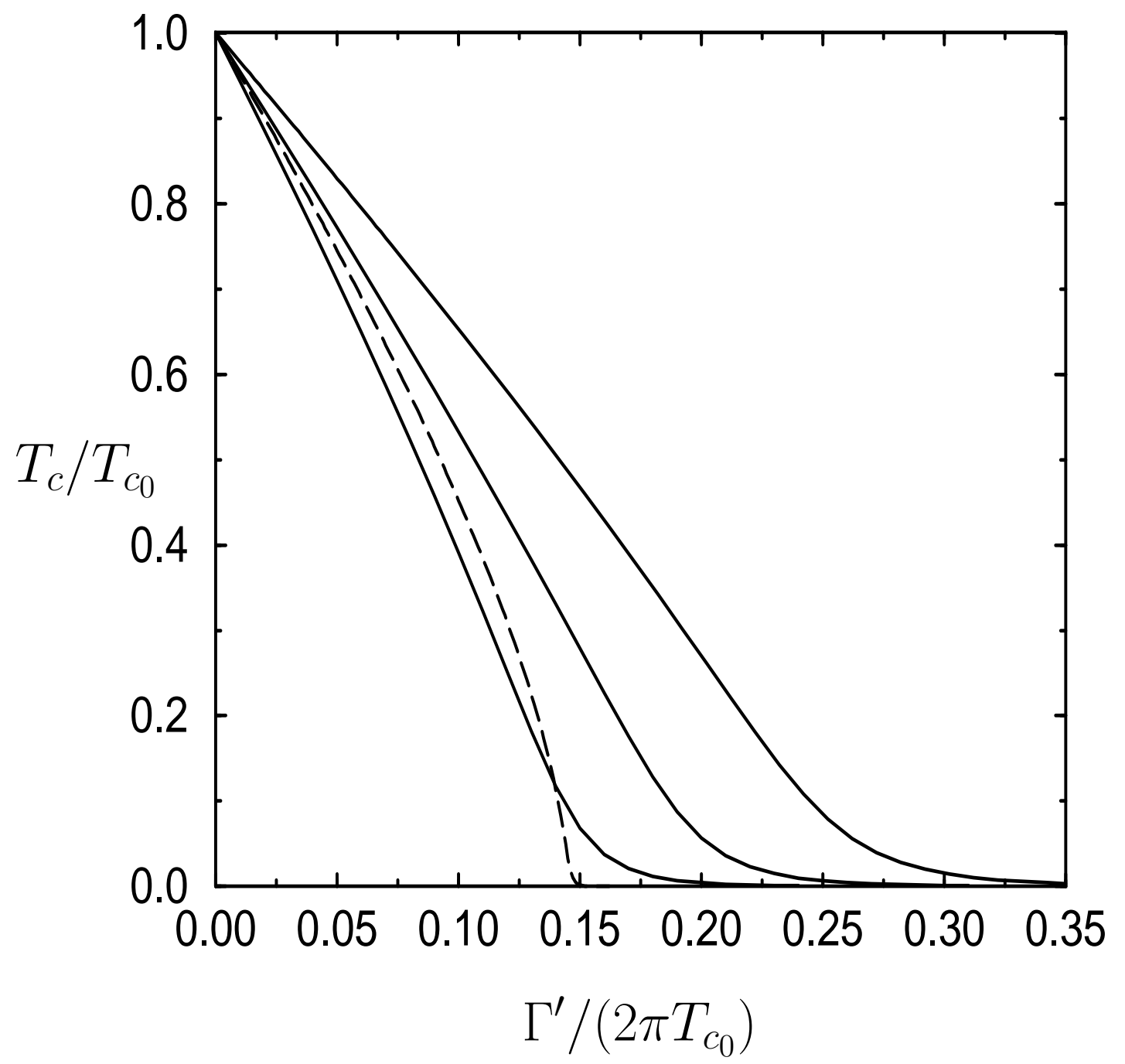

FIG. 10. Fig.6: 


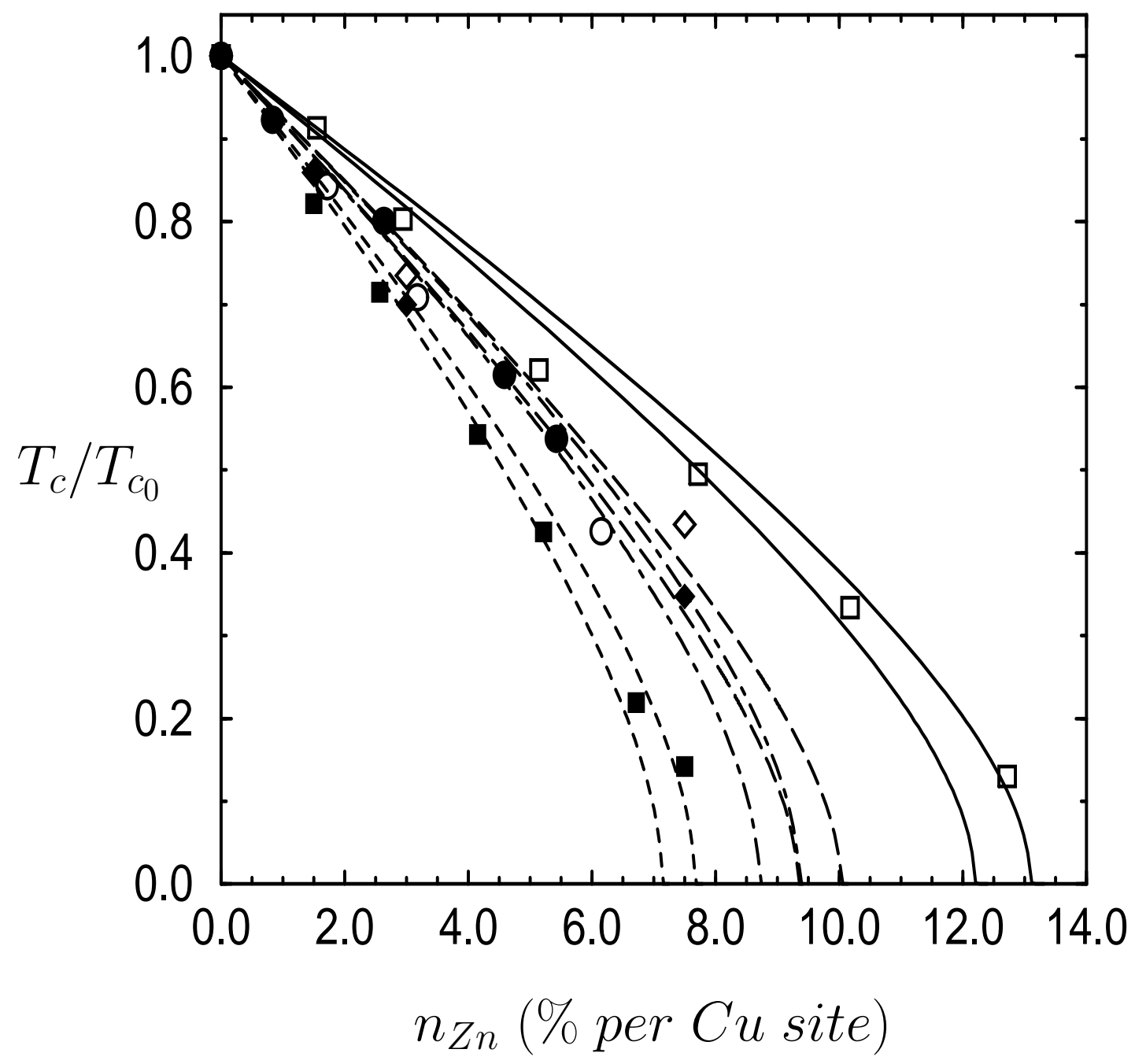

FIG. 11. Fig.7: 


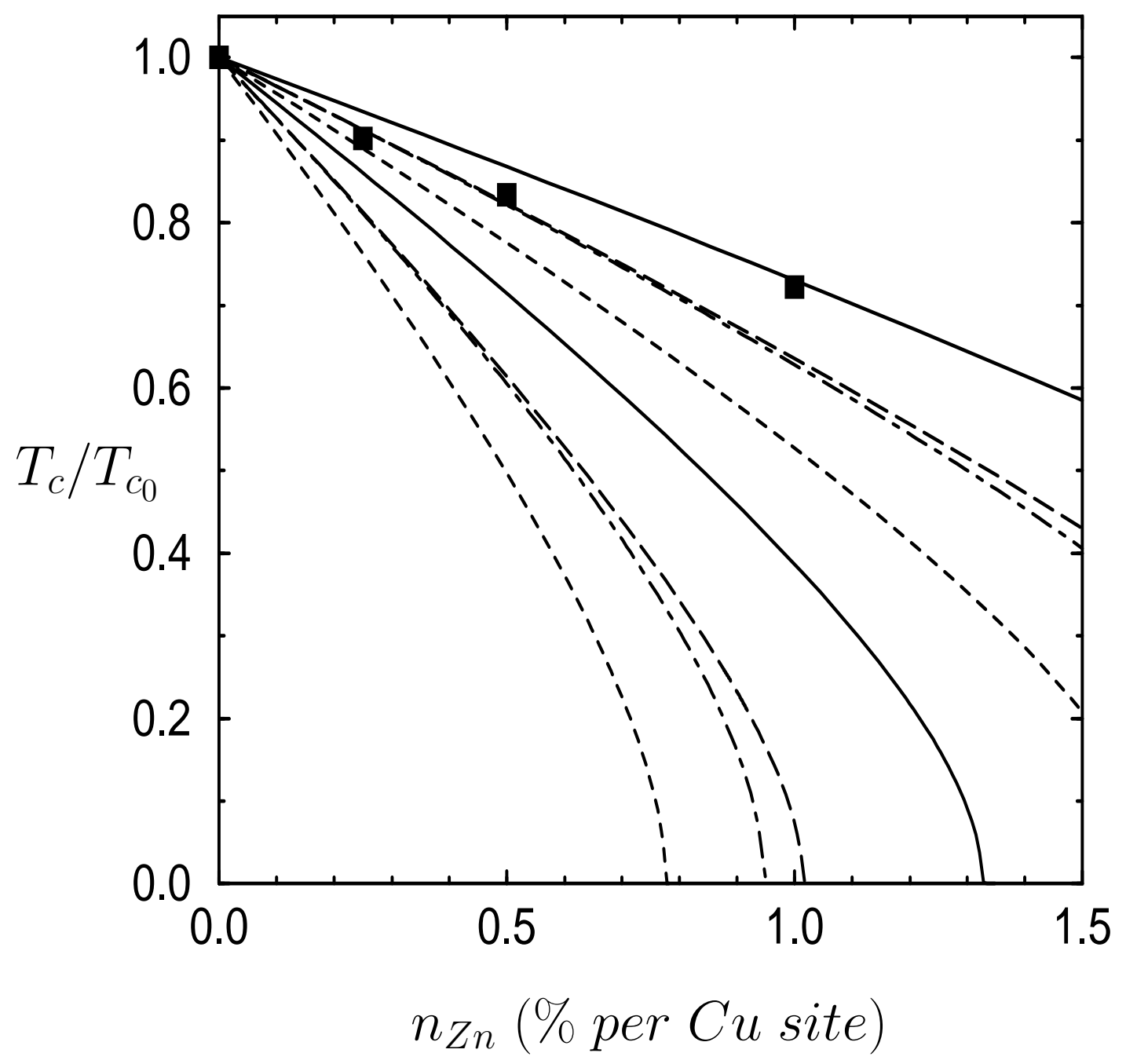

FIG. 12. Fig.8a: 


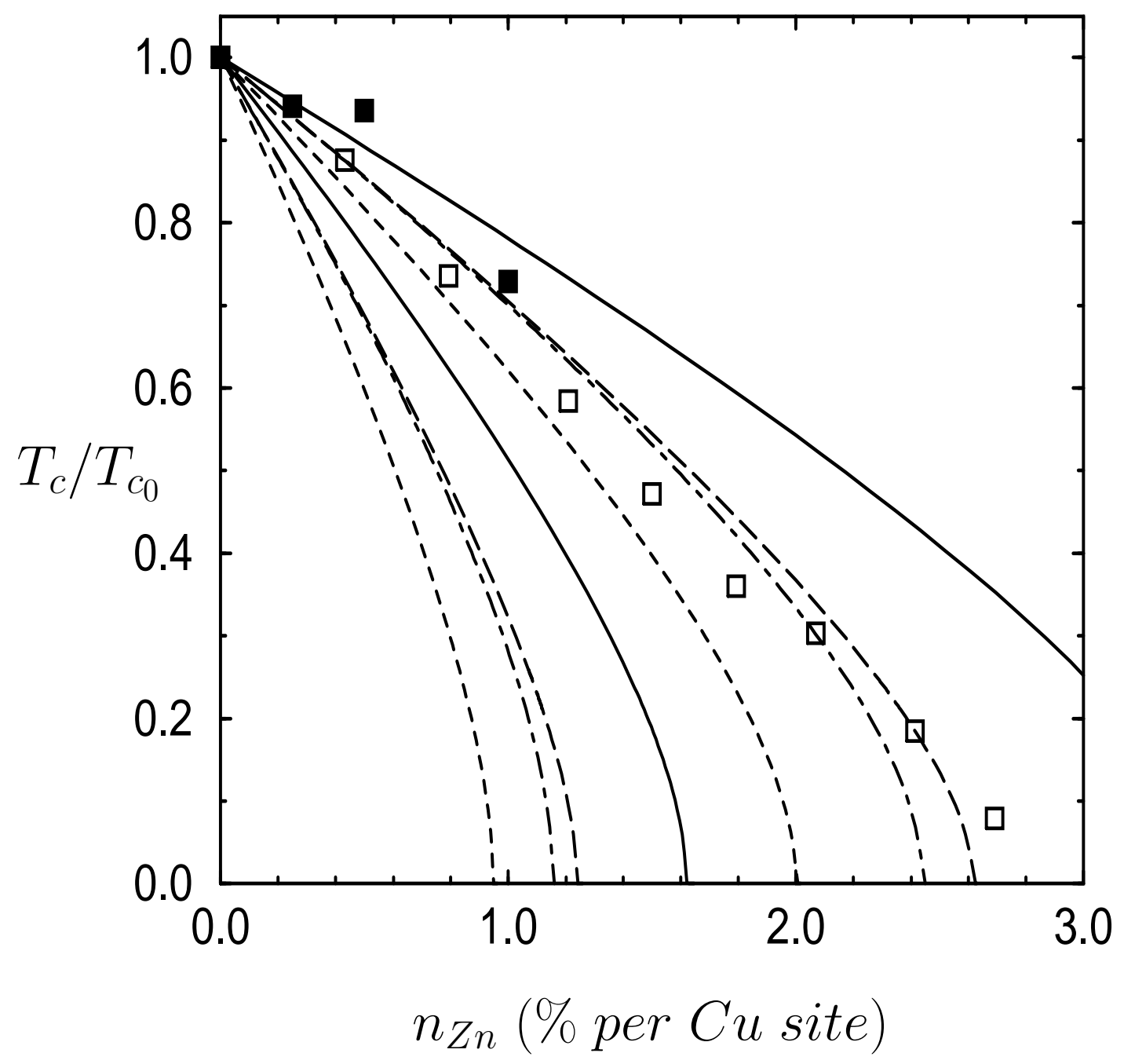

FIG. 13. Fig.8b: 


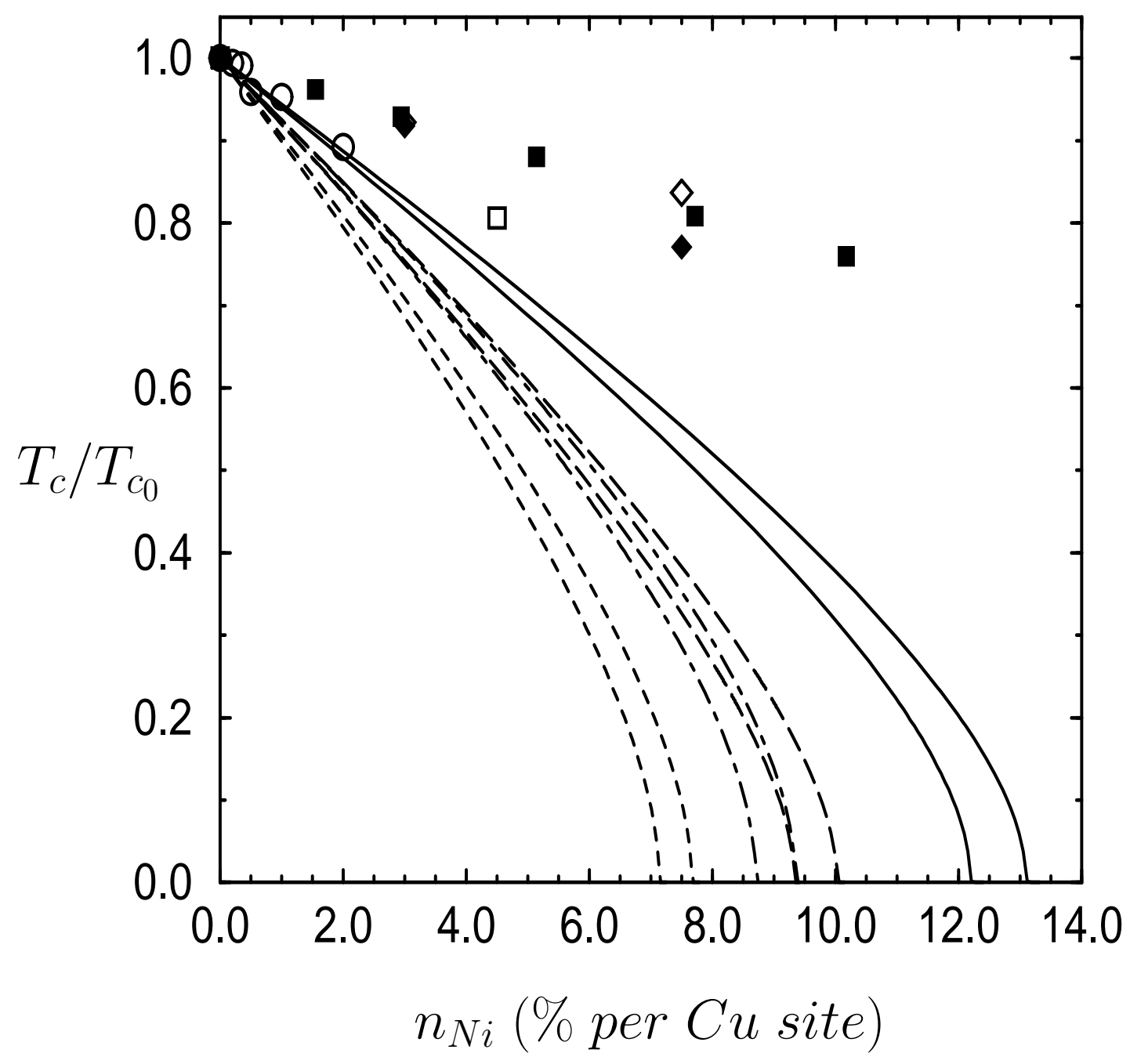

FIG. 14. Fig.9: 


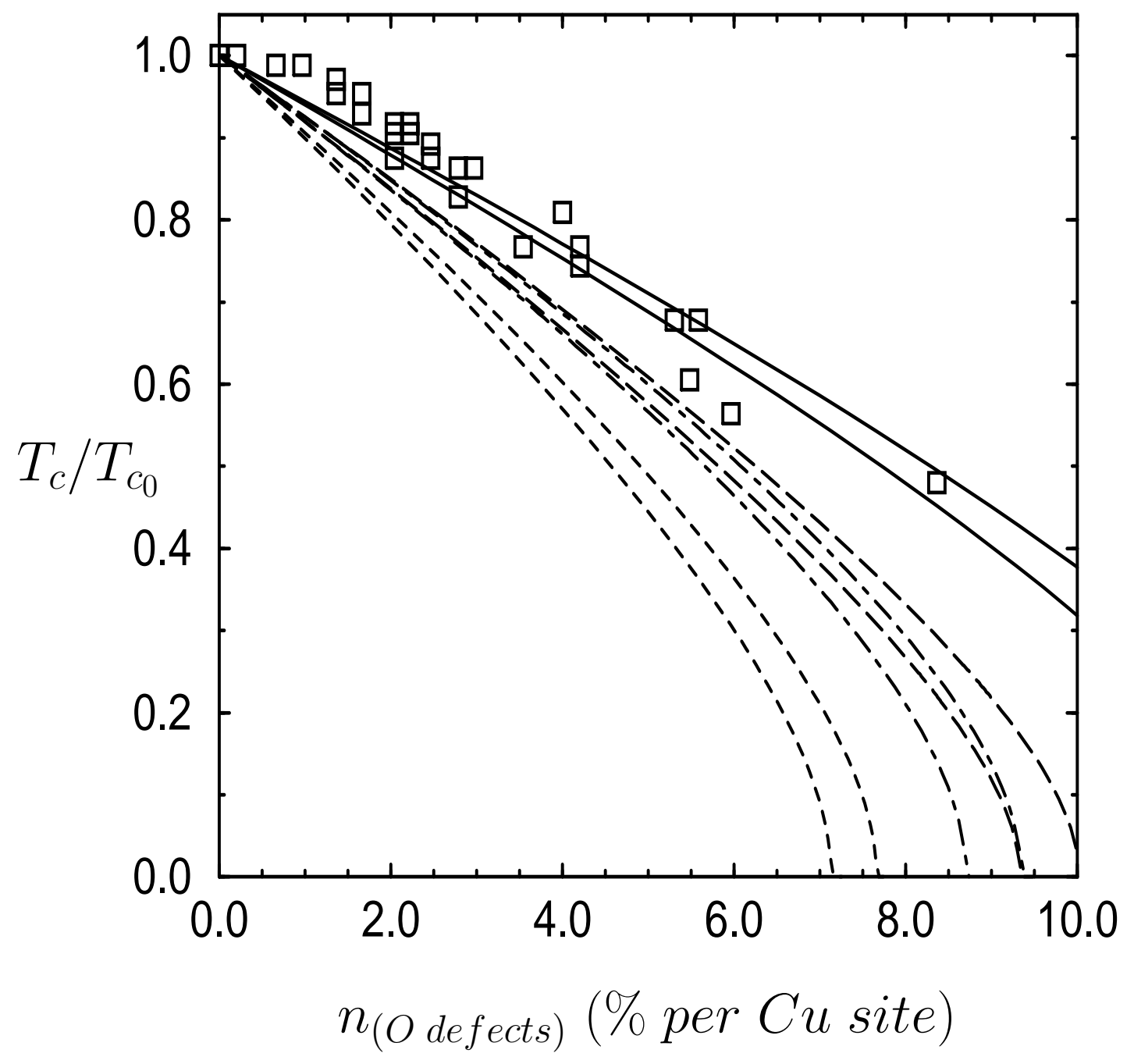

FIG. 15. Fig.10: 